\title{
Renormalization-Group Improvement of Effective Actions Beyond Summation of Leading Logarithms
}

\author{
M. R. Ahmady, ${ }^{a}$ V. Elias, ${ }^{b, c}$ D. G. C. McKeon, ${ }^{c}$ \\ A. Squires, ${ }^{a}$ and T. G. Steele ${ }^{d}$ \\ ${ }^{a}$ Department of Physics, \\ Mount Allison University, Sackville, NB E4L 1E6, Canada \\ ${ }^{b}$ Perimeter Institute for Theoretical Physics, \\ 35 King Street North, Waterloo, Ontario N2J 2W9, Canada \\ ${ }^{c}$ Department of Applied Mathematics, \\ The University of Western Ontario, London, Ontario N6A 5B7, Canada \\ ${ }^{d}$ Department of Physics and Engineering Physics, \\ University of Saskatchewan, Saskatoon, Saskatchewan S7N 5E2, Canada
}

\begin{abstract}
Invariance of the effective action under changes of the renormalization scale $\mu$ leads to relations between those (presumably calculated) terms independent of $\mu$ at a given order of perturbation theory and those higher order terms dependent on logarithms of $\mu$. This relationship leads to differential equations for a sequence of functions, the solutions of which give closed form expressions for the sum of all leading logs, next to leading logs and subsequent subleading logarithmic contributions to the effective action. The renormalization group is thus shown to provide information about a model beyond the scale dependence of the model's couplings and masses. This procedure is illustrated using the $\phi_{6}^{3}$ model and Yang-Mills theory. In the latter instance, it is also shown by using a modified summation procedure that the $\mu$ dependence of the effective action resides solely in a multiplicative factor of $g^{2}(\mu)$ (the running coupling). This approach is also shown to lead to a novel expansion for the running coupling in terms of the one-loop coupling that does not require an order-by-order redefinition of the scale factor $\Lambda_{Q C D}$. Finally, logarithmic contributions of the instanton size to the effective action of an SU(2) gauge theory are summed, allowing a determination of the asymptotic dependence on the instanton size $\rho$ as $\rho$ goes to infinity to all orders in the $\mathrm{SU}(2)$ coupling constant.
\end{abstract}

\section{Introduction}

It is well understood that self-interactions in quantum field theory can serve to rescale (renormalize) the parameters that characterize the theory. Indeed, renormalization is usually viewed as necessary to eliminate divergences which arise when physical processes are computed. In the course of this renormalization, a mass scale $\mu$ inevitably arises, apparently rendering the results of any computation inherently ambiguous. The effect is illusory $[1,2]$; one can compensate for changes in $\mu$ by concomitant changes in the couplings, masses and field strengths characterizing the theory under consideration. Indeed, it is this observation, which ultimately implies that all physical quantities in the theory should be independent of $\mu$, which leads to the renormalizationgroup (RG) equation. When calculating quantities to only a finite order in perturbation theory, however, there remains a residual dependence on $\mu$. 
The formal solution of the RG equation results in a dependence of the couplings and masses on the parameter $\mu$ in a manner fixed by the so-called RG functions. Generally speaking, these functions are determined by the relationship between the renormalized and (presumably infinite) bare couplings in the context of some regulating scheme, such as dimensional regularization [3]. However, when one uses $\zeta$-function regularization [4] or its multiloop generalization, operator regularization [5], no explicit divergences appear when the regulating parameter vanishes; explicit renormalization is not required to excise infinities in these schemes. A mass scale $\mu$ nevertheless arises in the course of using such schemes. Hence, in this approach one is forced to determine the RG functions by direct examination of the $\mu$-dependence of the effective action. This approach can in principle also be used to determine the RG functions when one is using dimensional regularization, though in practice the RG functions in dimensional regularization are extracted by considering the relationship between bare and renormalized quantities. Such an approach has been explicitly developed to two-loop order in two different massive scalar field theories, one with a cubic self-interaction coupling in six dimensions [6], and one with the usual quartic self-interaction coupling in four dimensions [7].

However, the RG equation can do more than just provide the usual mass scale dependence to the couplings and masses in a given model. Consistency conditions arise which relate logarithms occurring in higher loop calculations to non-logarithmic contributions that occur at lower orders. In dimensional regularization, these correspond to relationships between coefficients of poles appearing in the equation relating the bare and renormalized couplings $[2,8]$. Such consistency conditions also constitute relations between the coefficients of logarithms appearing in the effective action [9], in both operator regularization and dimensional regularization.

In the present paper, we exploit these relationships to "RG-improve" such effective actions via explicit summation of leading and successively subleading logarithms accessible from the RG-equation to all orders in perturbation theory. Such an approach has already been applied to a number of $\overline{M S}$ perturbative processes [10] as well as to the relationship between bare and renormalized coupling constants in the context of dimensional regularization [8]. In Section 2, we continue the work of ref. [9], in which leading logarithms were summed to all orders, by summing to all orders in perturbation theory the next-to-leading-logarithmic radiative corrections to the classical action for a massive scalar field theory in six dimensions with trilinear self-interaction coupling $\left(\phi_{6}^{3}\right)$. This simple field theoretical example is particularly useful in that the effective action involves separate kinetic, mass and self-interaction radiative corrections. The procedures delineated in Section 2 can easily be extended to all-orders summation of subsequent nonleading sets of logarithms, once appropriate perturbative calculations beyond those of ref. [6] become available. The procedure used is related to one employed in [11, 12] in the context of the effective potential for a massive $\phi_{4}^{4}$ model, as well as that employed in ref. [13] to sum the leading-logarithm contributions to the effective action of QED.

In Section 3, we use an approach first delineated in the Appendix to ref. [10] to find an allorders solution to the RG-equation for the kinetic term of the $\phi_{6}^{3}$ theory via a restructured version of the series for this kinetic term obtained in Section 2. The consistency of this closed-form expression for the kinetic term with the series expression extracted in Section 2 is demonstrated in Appendix A.

In Section 4 we first apply the methods of Section 2 to obtain the all-orders summation of leading and the first three subsequent subleading sets of logarithmic corrections to the effective action for Yang-Mills theory. We then apply the methods developed in Section 3 to an appropriately restructured series for this effective action. For this latter approach, we are able to determine in full the coefficient of $F_{\mu \nu a} F^{\mu \nu a}$ if the $\beta$-function is known to all orders and the coefficient is known for a particular value of $\mu$. Such an approach is particularly relevant for $N=1$ supersymmetric Yang-Mills theory, for which the $\beta$-function is entirely known $[14,15]$. Moreover, we are also able to demonstrate that all the $\mu$-dependence of this coefficient is necessarily proportional to the running gauge coupling constant $g^{2}(\mu)$. 
In Section 5 we demonstrate how a direct comparison of the two approaches delineated in Section 4 can be employed to extract an alternate series solution for the running coupling constant. As a check on our approach, a derivation of this same series based upon explicit use of $\mathrm{RG}$ invariance is presented in Appendix B. For $\mathrm{SU}(\mathrm{N})$ vector-like gauge theories, such as $\mathrm{QCD}$, the series we obtain enables us to express the four-loop running couplant as a series in which the naïve one-loop running couplant surprisingly appears as the expansion parameter. We conclude Section 5 by discussing in detail the departure of this series in the infrared region from the true running couplant's behaviour, as determined from explicit integration over the couplant's $\beta$-function. Such a departure is shown to reflect the disparity between the Landau singularity in the one-loop couplant, which is the series expansion parameter, and the Landau singularity characterising evolution via the full four-loop order $\beta$-function.

Finally, in Section 6 we consider how the instanton contribution to the effective Lagrangian of an $\mathrm{SU}(2)$ gauge theory is affected by all-orders summation of logarithms of the instanton size. Since the instanton size $\rho$ only appears in logarithmic contributions to the perturbative series in the $\mathrm{SU}(2)$ gauge coupling constant, it is possible to sum arbitrarily-subleading logarithms in order to obtain asymptotic dependence on $\rho$ (as $\rho$ becomes large) to all orders in the coupling constant. We find that the asymptotic behaviour of the integral over instanton size retains essentially the same infrared divergence previously extracted by 't Hooft [16] from the oneloop contribution, with only a slight logarithmic modification arising from the summation of next-to-leading logs. The summation of subsequent logarithms in the perturbative series (i.e. logarithms other than leading and next-to-leading) are shown not to contribute to the asymptotic $\rho$-dependence in the large- $\rho$ limit. 


\section{RG-Resummation of the $\phi_{6}^{3}$ Effective Action}

In ref. [6], the leading logarithms within the effective action for a scalar field $(f)$ theory with a trilinear self-coupling in six dimensions are computed. These leading logarithms are summed in ref. [9]. The classical action for such a theory in Euclidean space is just

$$
\Gamma_{0}[f(x)]=-\frac{1}{2} f(x) \partial^{2} f(x)+\frac{m^{2}}{2} f^{2}(x)+\frac{\lambda f^{3}(x)}{3 !} .
$$

Radiative corrections to eq. (2.1) leave us with an effective action of the form

$$
\Gamma[f]=-\frac{1}{2} f \partial^{2} f A(\lambda, L)+\frac{m^{2}}{2} f^{2} B(\lambda, L)+\frac{\lambda f^{3}}{3 !} C(\lambda, L)
$$

with $L \equiv \log \left(m^{2} / \mu^{2}\right)$ and with

$$
\begin{aligned}
& A(\lambda, L)=\sum_{n=0}^{\infty} \sum_{m=n}^{\infty} a_{m, n} \lambda^{2 m} L^{n}, \\
& B(\lambda, L)=\sum_{n=0}^{\infty} \sum_{m=n}^{\infty} b_{m, n} \lambda^{2 m} L^{n}, \\
& C(\lambda, L)=\sum_{n=0}^{\infty} \sum_{m=n}^{\infty} c_{m, n} \lambda^{2 m} L^{n} .
\end{aligned}
$$

Since the effective action (2.2) must be independent of $\mu$, we have the RGE

$$
\mu \frac{d \Gamma}{d \mu}=0=\left(\mu \frac{\partial}{\partial \mu}+\beta(\lambda) \frac{\partial}{\partial \lambda}-\gamma_{m}(\lambda) m^{2} \frac{\partial}{\partial m^{2}}-\gamma_{\Gamma}(\lambda) \int d^{4} x^{\prime} f\left(x^{\prime}\right) \frac{\delta}{\delta f\left(x^{\prime}\right)}\right) \Gamma,
$$

where the RG functions within eq. (2.6) are defined as follows:

$$
\begin{gathered}
\beta(\lambda) \equiv \mu \frac{d \lambda}{d \mu}=\sum_{n=1}^{\infty} B_{2 n+1} \lambda^{2 n+1} \\
-\gamma_{m}(\lambda) m^{2} \equiv \mu \frac{d m^{2}}{d \mu}=-m^{2} \sum_{n=1}^{\infty} G_{2 n} \lambda^{2 n} \\
-\gamma_{\Gamma}(\lambda) f \equiv \mu \frac{d f}{d \mu}=-f \sum_{n=1}^{\infty} D_{2 n} \lambda^{2 n}
\end{gathered}
$$

Noting for an arbitrary function $F(\lambda, L)$ that $\mu \frac{\partial}{\partial \mu} F(\lambda, L)=-2 \frac{\partial}{\partial L} F(\lambda, L)$ and that $m^{2} \frac{\partial}{\partial m^{2}} F(\lambda, L)=$ $\frac{\partial}{\partial L} F(\lambda, L)$, we substitute eq. (2.2) into eq. (2.6) to obtain the following RG-equations for $A(\lambda, L)$, $B(\lambda, L)$ and $C(\lambda, L)$ :

$$
\begin{gathered}
{\left[-\left(2+\gamma_{m}(\lambda)\right) \frac{\partial}{\partial L}+\beta(\lambda) \frac{\partial}{\partial \lambda}-2 \gamma_{\Gamma}(\lambda)\right] A(\lambda, L)=0} \\
{\left[-\left(2+\gamma_{m}(\lambda)\right) \frac{\partial}{\partial L}+\beta(\lambda) \frac{\partial}{\partial \lambda}-\left(2 \gamma_{\Gamma}(\lambda)+\gamma_{m}(\lambda)\right)\right] B(\lambda, L)=0} \\
{\left[-\left(2+\gamma_{m}(\lambda)\right) \frac{\partial}{\partial L}+\beta(\lambda) \frac{\partial}{\partial \lambda}+\left(\frac{\beta(\lambda)}{\lambda}-3 \gamma_{\Gamma}(\lambda)\right)\right] C(\lambda, L)=0 .}
\end{gathered}
$$


Upon substitution of eq. (2.3) and eqs. (2.7-2.9) into eq. (2.10), we find the aggregate coefficients of $\lambda^{2 n} L^{n-1}$ and $\lambda^{2 n} L^{n-2}$ on the left hand side of eq. (2.10) respectively vanish provided the following recursion relations are upheld:

$$
\begin{gathered}
-2 n a_{n, n}+\left[2(n-1) B_{3}-2 D_{2}\right] a_{n-1, n-1}=0, \\
-2(n-1) a_{n, n-1}+\left[2(n-1) B_{3}-2 D_{2}\right] a_{n-1, n-2} \\
-(n-1) G_{2} a_{n-1, n-1}+\left[2(n-2) B_{5}-2 D_{4}\right] a_{n-2, n-2}=0 .
\end{gathered}
$$

Substitution of eq. (2.4) and eqs. (2.7-2.9) into eq. (2.11) also yields recursion relations for $b_{n, m}$ :

$$
\begin{gathered}
-2 n b_{n, n}+\left[2(n-1) B_{3}-2 D_{2}-G_{2}\right] b_{n-1, n-1}=0, \\
-2(n-1) b_{n, n-1}+\left[2(n-1) B_{3}-2 D_{2}-G_{2}\right] b_{n-1, n-2} \\
-(n-1) G_{2} b_{n-1, n-1}+\left[2(n-2) B_{5}-2 D_{4}-G_{4}\right] b_{n-2, n-2}=0,
\end{gathered}
$$

and similar substitution of eq. (2.5) and eqs. (2.7-2.9) into eq. (2.12) yields the following recursion relations for $c_{n, m}$ :

$$
\begin{gathered}
-2 n c_{n, n}+\left[(2 n-1) B_{3}-3 D_{2}\right] c_{n-1, n-1}=0, \\
-2(n-1) c_{n, n-1}+\left[(2 n-1) B_{3}-3 D_{2}\right] c_{n-1, n-2} \\
-(n-1) G_{2} c_{n-1, n-1}+\left[(2 n-3) B_{5}-3 D_{4}\right] c_{n-2, n-2}=0 .
\end{gathered}
$$

An explicit perturbative computation [7] produces the following values for the leading two orders of coefficients in $(2.3-2.5)\left[k \equiv 1 /(4 \pi)^{3}\right]$ :

$$
\begin{gathered}
a_{0,0}=b_{0,0}=c_{0,0} \equiv 1, \\
a_{1,0}=0, \quad b_{1,0}=k / 2, \quad c_{1,0}=0, \\
a_{1,1}=-k / 12, \quad b_{1,1}=-k / 2, \quad c_{1,1}=-k / 2, \\
a_{2,1}=k^{2} / 216, \quad b_{2,1}=-43 k^{2} / 48, \quad c_{2,1}=-13 k^{2} / 48, \\
a_{2,2}=5 k^{2} / 144, \quad b_{2,2}=5 k^{2} / 16, \quad c_{2,2}=5 k^{2} / 16 .
\end{gathered}
$$

If we choose $n=1$ in eqs. (2.13), (2.15) and (2.17), we find from the coefficients (2.19) and (2.21) that the lead terms of the RG-functions (2.7-2.9) are

$$
B_{3}=-3 k / 4, \quad G_{2}=5 k / 6, \quad D_{2}=k / 12 .
$$

Indeed, these results are sufficient to determine the coefficients (2.23) by setting $n=2$ in eqs. (2.13), (2.15) and (2.17). Similarly one can substitute coefficients (2.19-2.22) and leading terms (2.24) into the $n=2$ versions of the recursion relations (2.14), (2.16) and (2.18) to obtain the next-to-leading contributions to the RG functions (2.7-2.9):

$$
B_{5}=-125 k^{2} / 144, \quad G_{4}=97 k^{2} / 108, \quad D_{4}=13 k^{2} / 432 .
$$

The RG-function results (2.24) and (2.25) plus the recursion relations (2.13-18) permit one to sum the leading and next-to-leading logarithmic contributions to $A, B$, and $C$ to all orders of perturbation theory. The leading-logarithm (LL) contribution to $A$, as given by (2.3), is just

$$
\begin{aligned}
(A(\lambda, L))_{L L} & =a_{0,0}+a_{1,1} \lambda^{2} L+a_{2,2}\left(\lambda^{2} L\right)^{2}+a_{3,3}\left(\lambda^{2} L\right)^{3}+\ldots \\
& =\sum_{n=0}^{\infty} a_{n, n}\left(\lambda^{2} L\right)^{n} \equiv R_{0}\left(\lambda^{2} L\right) .
\end{aligned}
$$


If we define $u \equiv \lambda^{2} L$ and multiply the recursion relation (2.13) by $u^{n-1}$ and then sum over $n$ from $n=1$ to $\infty$, we obtain the differential equation

$$
\left(1-B_{3} u\right) R_{0}^{\prime}(u)+D_{2} R_{0}(u)=0
$$

with initial condition $R_{0}(0)=a_{0,0}=1$. The solution to this differential equation is

$$
R_{0}\left(\lambda^{2} L\right)=\left(1-B_{3} \lambda^{2} L\right)^{D_{2} / B_{3}}=\left(1+3 \lambda^{2} L / 256 \pi^{3}\right)^{-1 / 9} .
$$

To find $L L$ contributions to $B$ and $C$,

$$
\begin{aligned}
& (B(\lambda, L))_{L L}=\sum_{n=0}^{\infty} b_{n, n}\left(\lambda^{2} L\right)^{n} \equiv S_{0}\left(\lambda^{2} L\right), \\
& (C(\lambda, L))_{L L}=\sum_{n=0}^{\infty} c_{n, n}\left(\lambda^{2} L\right)^{n} \equiv T_{0}\left(\lambda^{2} L\right),
\end{aligned}
$$

one may apply exactly the same procedure as above to obtain differential equations for $S_{0}$ and $T_{0}$ from the recursion relations (2.15) and (2.17):

$$
\begin{aligned}
& \left(1-B_{3} u\right) S_{0}^{\prime}(u)+\left(D_{2}+G_{2} / 2\right) S_{0}(u)=0 \\
& \left(1-B_{3} u\right) T_{0}^{\prime}+\left(3 D_{2} / 2-B_{3} / 2\right) T_{0}(u)=0 .
\end{aligned}
$$

Since $S_{0}(0)=b_{0,0}=1$ and $T_{0}(0)=c_{0,0}=1$, we easily find the solutions

$$
\begin{aligned}
& S_{0}\left(\lambda^{2} L\right)=\left(1-B_{3} \lambda^{2} L\right)^{\left(D_{2}+\frac{1}{2} G_{2}\right) / B_{3}}=\left(1+3 \lambda^{2} L / 256 \pi^{3}\right)^{-2 / 3}, \\
& T_{0}\left(\lambda^{2} L\right)=\left(1-B_{3} \lambda^{2} L\right)^{\left(3 D_{2}-B_{3}\right) / 2 B_{3}}=\left(1+3 \lambda^{2} L / 256 \pi^{3}\right)^{-2 / 3} .
\end{aligned}
$$

It is curious that the summations of LL contributions to $B$ and $C$ are identical.

The recursion relations (2.14), (2.16), and (2.18) may be similarly employed to determine the next-to-leading logarithm (NLL) contributions to $A(\lambda, L), B(\lambda, L)$ and $C(\lambda, L)$, as given by eqs. (2.3-2.5):

$$
\begin{aligned}
{[A(\lambda, L)]_{N L L} } & =a_{1,0} \lambda^{2}+a_{2,1} \lambda^{4} L+a_{3,2} \lambda^{6} L^{2}+\ldots \\
& =\lambda^{2} \sum_{n=1}^{\infty} a_{n, n-1}\left(\lambda^{2} L\right)^{n-1} \equiv \lambda^{2} R_{1}\left(\lambda^{2} L\right), \\
{[B(\lambda, L)]_{N L L} } & =\lambda^{2} \sum_{n=1}^{\infty} b_{n, n-1}\left(\lambda^{2} L\right)^{n-1} \equiv \lambda^{2} S_{1}\left(\lambda^{2} L\right), \\
{[C(\lambda, L)]_{N L L} } & =\lambda^{2} \sum_{n=1}^{\infty} c_{n, n-1}\left(\lambda^{2} L\right)^{n-1} \equiv \lambda^{2} T_{1}\left(\lambda^{2} L\right) .
\end{aligned}
$$

Upon multiplication of the recursion relations (2.14), (2.16) and (2.18) by $u^{n-2}$ followed by summation from $n=2$ to $\infty$, the series definitions $(2.26),(2.29)$, and (2.30) for $R_{0}(u), S_{0}(u)$, and $T_{0}(u)$, and the definitions $(2.35-2.37)$ for $R_{1}(u), S_{1}(u)$, and $T_{1}(u)$ lead to the following linear first order differential equations for the latter three quantities:

$$
\begin{gathered}
\left(1-B_{3} u\right) \frac{d R_{1}(u)}{d u}+\left(D_{2}-B_{3}\right) R_{1}(u)=\left[\left(B_{5} u-\frac{G_{2}}{2}\right) \frac{d}{d u}-D_{4}\right] R_{0}(u), \\
\left(1-B_{3} u\right) \frac{d S_{1}(u)}{d u}+\left(D_{2}+\frac{G_{2}}{2}-B_{3}\right) S_{1}(u)=\left[\left(B_{5} u-\frac{G_{2}}{2}\right) \frac{d}{d u}-D_{4}-\frac{G_{4}}{2}\right] S_{0}(u),
\end{gathered}
$$




$$
\left(1-B_{3} u\right) \frac{d T_{1}(u)}{d u}+\left(\frac{3 D_{2}}{2}-B_{3}\right) T_{1}(u)=\left[\left(B_{5} u-\frac{G_{2}}{2}\right) \frac{d}{d u}-\frac{3 D_{4}}{2}+\frac{B_{5}}{2}\right] T_{0}(u) .
$$

Given the explicit solutions (2.28), (2.33), (2.34) already obtained for $R_{0}, S_{0}$, and $T_{0}$, and given the initial conditions $R_{1}(0)=a_{1,0}=0, S_{1}(0)=b_{1,0}=k / 2, T_{1,0}=c_{1,0}=0$, we obtain the following solutions to eqs. $(2.38-2.40)$ :

$$
\begin{aligned}
& R_{1}(u)=\left[\frac{D_{4}}{B_{3}}-\frac{D_{2} B_{5}}{B_{3}^{2}}\right]\left(1-B_{3} u\right)^{D_{2} / B_{3}} \\
& +\frac{\left[a_{1,0}-\left[\frac{D_{4}}{B_{3}}-\frac{D_{2} B_{5}}{B_{3}^{2}}\right]-\frac{D_{2}}{B_{3}}\left(\frac{G_{2}}{2}-\frac{B_{5}}{B_{3}}\right) \log \left(1-B_{3} u\right)\right]}{\left(1-B_{3} u\right)^{1-D_{2} / B_{3}}} \\
& =\frac{1}{(4 \pi)^{3}}\left\{\frac{43}{486}\left(1+\frac{3 u}{256 \pi^{3}}\right)^{-1 / 9}-\left[\frac{43}{486}+\frac{20}{243} \log \left(1+\frac{3 u}{256 \pi^{3}}\right)\right]\left(1+\frac{3 u}{256 \pi^{3}}\right)^{-10 / 9}\right\} \\
& S_{1}(u)=\left[-\frac{\left(2 D_{2}+G_{2}\right) B_{5}}{2 B_{3}^{2}}+\frac{\left(2 D_{4}+G_{4}\right)}{2 B_{3}}\right]\left(1-B_{3} u\right)^{\frac{2 D_{2}+G_{2}}{2 B_{3}}} \\
& +\left\{b_{1,0}-\left[\frac{\left(2 D_{4}+G_{4}\right)}{2 B_{3}}-\frac{\left(2 D_{2}+G_{2}\right)}{2 B_{3}^{2}} B_{5}\right]\right. \\
& \left.-\frac{\left(2 D_{2}+G_{2}\right)}{2 B_{3}}\left(\frac{G_{2}}{2}-\frac{B_{5}}{B_{3}}\right) \log \left(1-B_{3} u\right)\right\}\left(1-B_{3} u\right)^{\frac{2 D_{2}+G_{2}-2 B_{3}}{2 B_{3}}} \\
& =\frac{1}{(4 \pi)^{3}}\left\{\frac{43}{324}\left(1+\frac{3 u}{256 \pi^{3}}\right)^{-2 / 3}+\left[\frac{119}{324}-\frac{40}{81} \log \left(1+\frac{3 u}{256 \pi^{3}}\right)\right]\left(1+\frac{3 u}{256 \pi^{3}}\right)^{-5 / 3}\right\} \text {, } \\
& T_{1}(u)=\left[-\frac{\left(3 D_{2}-B_{3}\right)}{2 B_{3}^{2}} B_{5}+\frac{\left(3 D_{4}-B_{5}\right)}{2 B_{3}}\right]\left(1-B_{3} u\right)^{\frac{3 D_{2}-B_{3}}{2 B_{3}}} \\
& +\left\{c_{1,0}-\left[\frac{\left(3 D_{4}-B_{5}\right)}{2 B_{3}}-\frac{\left(3 D_{2}-B_{3}\right) B_{5}}{2 B_{3}^{2}}\right]\right. \\
& \left.-\frac{\left(3 D_{2}-B_{3}\right)}{2 B_{3}}\left(\frac{G_{2}}{2}-\frac{B_{5}}{B_{3}}\right) \log \left(1-B_{3} u\right)\right\}\left(1-B_{3} u\right)^{\frac{3\left(D_{2}-B_{3}\right)}{2 B_{3}}} \\
& =\frac{1}{(4 \pi)^{3}}\left\{\frac{43}{324}\left(1+\frac{3 u}{256 \pi^{3}}\right)^{-2 / 3}-\left[\frac{43}{324}+\frac{40}{81} \log \left(1+\frac{3 u}{256 \pi^{3}}\right)\right]\left(1+\frac{3 u}{256 \pi^{3}}\right)^{-5 / 3}\right\} \text {. }
\end{aligned}
$$

For the procedures delineated above to be extended to the summation of subsequent subleading logs, one must have perturbatively-calculated values of the coefficients $a_{k, 0}, b_{k, 0}, c_{k, 0}$ with $k \geq 3$. One is then able to utilize subsequent recursion relations devolving from the $R G E$ 's $(2.10-12)$ to obtain first-order differential equations for

$$
\left(\begin{array}{c}
R_{k}(u) \\
S_{k}(u) \\
T_{k}(u)
\end{array}\right)=\sum_{n=k}^{\infty}\left(\begin{array}{c}
a_{n, n-k} \\
b_{n, n-k} \\
c_{n, n-k}
\end{array}\right) u^{n-k}
$$

with known initial values $R_{k}(0)=a_{k, 0}, S_{k}(0)=b_{k, 0}, T_{k}(0)=c_{k, 0}$. Solutions of these differential equations for progressively larger values of $k$ allows one to calculate $A, B$ and $C$ as a succession 
of all-orders summations of successively-subleading logarithms

$$
\begin{gathered}
A(\lambda, L)=(A(\lambda, L))_{L L}+(A(\lambda, L))_{N L L}+\ldots=\sum_{k=0}^{\infty} \lambda^{2 k} R_{k}\left(\lambda^{2} L\right), \\
B(\lambda, L)=\sum_{k=0}^{\infty} \lambda^{2 k} S_{k}\left(\lambda^{2} L\right) \\
C(\lambda, L)=\sum_{k=o}^{\infty} \lambda^{2 k} T_{k}\left(\lambda^{2} L\right) .
\end{gathered}
$$




\section{Closed-Form All-Orders Solution to $A(\lambda, L)$}

An alternative formulation of the summation of logarithms may be obtained by expressing the summations (2.3-2.5) in the following form, in which the dependence on $\lambda$ and $L$ factorizes in each term:

$$
\begin{gathered}
\left(\begin{array}{l}
A(\lambda, L) \\
B(\lambda, L) \\
C(\lambda, L)
\end{array}\right)=\sum_{n=0}^{\infty}\left(\begin{array}{c}
\rho_{n}\left(\lambda^{2}\right) \\
\sigma_{n}\left(\lambda^{2}\right) \\
\tau_{n}\left(\lambda^{2}\right)
\end{array}\right) L^{n} \\
\left(\begin{array}{c}
\rho_{n}\left(\lambda^{2}\right) \\
\sigma_{n}\left(\lambda^{2}\right) \\
\tau_{n}\left(\lambda^{2}\right)
\end{array}\right)=\sum_{m=n}^{\infty}\left(\begin{array}{c}
a_{m, n} \\
b_{m, n} \\
c_{m, n}
\end{array}\right)\left(\lambda^{2}\right)^{m} .
\end{gathered}
$$

Such a restructuring is developed into a procedure for closed form summation in the Appendix to ref. [10]. We utilize this procedure in the present section to demonstrate how $A(\lambda, L)$ within eq. (3.1) may be expressed in closed form. If we substitute the expression (3.1) for $A(\lambda, L)$ into the $R G E(2.10)$, we obtain the recursion relation

$$
\left(2+\gamma_{m}(\lambda)\right)(n+1) \rho_{n+1}=\left(\beta(\lambda) \frac{\partial}{\partial \lambda}-2 \gamma_{\Gamma}(\lambda)\right) \rho_{n} .
$$

We now define

$$
\rho_{n}(\lambda) \equiv \exp \left[\int_{0}^{\lambda} \frac{2 \gamma_{\Gamma}(w)}{\beta(w)} d w\right] \tilde{\rho}_{n}(\lambda)
$$

and find from eq. (3.2) that

$$
\tilde{\rho}_{n+1}=\left(\frac{1}{n+1}\right)\left(\frac{1}{2+\gamma_{m}(\lambda)}\right) \beta(\lambda) \frac{d \tilde{\rho}_{n}}{d \lambda} .
$$

From eqs. (3.3) and (3.4) it is straightforward to show that

$$
\tilde{A}(\lambda, L) \equiv \sum_{n=0}^{\infty} \tilde{\rho}_{n}(\lambda) L^{n}=\exp \left[\frac{L \beta(\lambda)}{2+\gamma_{m}(\lambda)} \frac{d}{d \lambda}\right] \tilde{\rho}_{0}(\lambda) .
$$

It is convenient here to introduce a new variable $\eta$ such that

$$
\frac{\beta(\lambda)}{2+\gamma_{m}(\lambda)} \frac{d}{d \lambda} \equiv \frac{d}{d \eta}
$$

This relation between differential operators is upheld provided

$$
\eta(\lambda)=\int_{\lambda_{0}}^{\lambda} \frac{\left(2+\gamma_{m}(w)\right)}{\beta(w)} d w .
$$

Equation (3.7) may be understood as defining $\lambda$ as an implicit function of $\eta$ such that $f(\eta(\lambda))=$ $\lambda$. Upon substitution of the differential operator (3.6) into eq. (3.5), we see that

$$
\begin{aligned}
\tilde{A}(\lambda, L) & =\exp \left(L \frac{d}{d \eta}\right) \tilde{\rho}_{0}(f(\eta(\lambda))) \\
& =\tilde{\rho}_{0}(f(\eta(\lambda)+L)) .
\end{aligned}
$$


We then obtain from eqs. (3.2-3.5) and (3.8) the following closed form solution for $A(\lambda, L)$ :

$$
\begin{aligned}
A(\lambda, L) & =\exp \left[\int_{0}^{\lambda} \frac{2 \gamma_{\Gamma}(w)}{\beta(w)} d w\right] \tilde{A}(\lambda, L) \\
& =\exp \left[\int_{0}^{\lambda} \frac{2 \gamma_{\Gamma}(w)}{\beta(w)} d w\right] \tilde{\rho}_{0}(f(\eta(\lambda)+L)) \\
& =\exp \left[-\int_{\lambda}^{f(\eta(\lambda)+L)} \frac{2 \gamma_{\Gamma}(w)}{\beta(w)} d w\right] \rho_{0}(f(\eta(\lambda)+L)) .
\end{aligned}
$$

The employment of such an all-orders solution, of course, rests upon complete knowledge of the function $\rho_{0}(\lambda)$, hence complete knowledge of the coefficients $a_{m, 0}$ for all $m$ (eq. (3.1)), as well as knowledge of the inverse function $f$ of $\eta(\lambda)$, as defined by eq. (3.7). For this inverse function, it is useful to note that knowledge of coefficients $a_{n, 0}, b_{n, 0}, c_{n, 0}$ is sufficient to determine the $n^{\text {th }}$-order coefficients of the $\beta$ and $\gamma$-functions (2.7-2.9) appearing in eqs. (3.7) and (3.9); this is evident for the $n=2$ case in the derivation of the coefficients (2.24) and (2.25) for these functions.

To illustrate how the solution (3.9) can actually be of use, we work with just leading order expressions $\beta(\lambda)=B_{3} \lambda^{3}, \gamma_{m}(\lambda)=G_{2} \lambda^{2}$, and $\gamma_{\Gamma}(\lambda)=D_{2} \lambda^{2}, \rho_{0}=a_{0,0}=1$, and we find from eq. (3.7) that

$$
-2 B_{3} \eta(\lambda)=\frac{2}{\lambda^{2}}-G_{2} \log \frac{\lambda^{2}}{\lambda_{0}^{2}}-\frac{2}{\lambda_{0}^{2}} .
$$

To solve for $\lambda=f(\eta(\lambda))$, we define $W \equiv 2 /\left(G_{2} \lambda^{2}\right)$ and find from eq. (3.10) that

$$
W+\log W=-\frac{2 B_{3} \eta}{G_{2}}+\frac{2}{G_{2} \lambda_{0}^{2}}+\log \left(\frac{2}{G_{2} \lambda_{0}^{2}}\right)
$$

or

$$
W e^{W}=\left[\frac{2}{G_{2} \lambda_{0}^{2}} \exp \left[\frac{2}{G_{2} \lambda_{0}^{2}}-\frac{2 B_{3} \eta}{G_{2}}\right]\right] .
$$

The relation $W(\xi) e^{W(\xi)}=\xi$ defines the Lambert W-function implicitly [17], in which case

$$
\lambda=f(\eta)=\left(\frac{2}{G_{2} W\left[\frac{2}{G_{2} \lambda_{0}^{2}} \exp \left(\frac{2}{G_{2} \lambda_{0}^{2}}-\frac{2 B_{3} \eta}{G_{2}}\right)\right]}\right)^{1 / 2} .
$$

We substitute $f(\eta+L)$, as defined by eq. (3.13), directly into eq. (3.9) to obtain

$$
\begin{aligned}
A(\lambda, L) & =\exp \left[-\int_{\lambda}^{f(\eta+L)} \frac{2 D_{2} w^{2}}{B_{3} w^{3}} d w\right] \cdot 1 \\
& =\left[\frac{G_{2} \lambda^{2} W\left[\frac{2}{G_{2} \lambda_{0}^{2}} \exp \left(\frac{2}{G_{2} \lambda_{0}^{2}}-\frac{2 B_{3}(\eta+L)}{G_{2}}\right)\right]}{2}\right]^{D_{2} / B_{3}} .
\end{aligned}
$$

We then substitute eq. (3.10) into the final line of eq. (3.14) to obtain

$$
A(\lambda, L)=\left[\frac{G_{2} \lambda^{2}}{2} W\left[\frac{2}{G_{2} \lambda^{2}} \exp \left(\frac{2}{G_{2} \lambda^{2}}-\frac{2 B_{3} L}{G_{2}}\right)\right]\right]^{D_{2} / B_{3}} .
$$

Eq.

(3.15) is a closed form solution to the RGE (2.10) based upon the premise that $B(\lambda), \gamma_{m}(\lambda)$ 
and $\gamma_{\Gamma}(\lambda)$ are given entirely by their leading order terms: $B_{3} \lambda^{3}, G_{2} \lambda^{2}$, and $D_{2} \lambda^{2}$, respectively. In Appendix A, we compare eq. (3.15) with the expression

$$
A(\lambda, L) \rightarrow R_{0}\left(\lambda^{2} L\right)=\left(1-B_{3} \lambda^{2} L\right)^{D_{2} / B_{3}}
$$

derived from equivalent lowest-order assumptions in the previous section. Specifically, we show that eqs. (3.15) and (3.16) differ at most by $\mathcal{O}\left(\lambda^{4}\right)$, as expected.'

\footnotetext{
${ }^{1}$ If they differed by $\mathcal{O}\left(\lambda^{2}\right)$, then $B_{3}$ in eq. (3.16) could be replaced by a new constant $B_{3}^{\prime}$ to eliminate the $\mathcal{O}\left(\lambda^{2}\right)$ discrepancy, in which case eq. (3.15) would not be a "best fit" to the expression (3.16).
} 


\section{RG-Resummation of the Yang-Mills Effective Action}

The analysis of Sections 2 and 3 is also applicable to Yang-Mills theory. If we employ background field quantization [18], then the renormalized effective action for Yang-Mills theory involves the following gauge-invariant form

$$
\Gamma[A]=-\frac{1}{4} F_{\mu \nu}^{a} F^{\mu \nu a} S\left[L, g^{2}\right]
$$

where in configuration space

$$
F_{\mu \nu}^{a}=\partial_{\mu} A_{\nu}^{a}-\partial_{\nu} A_{\mu}^{a}+g f^{a b c} A_{\mu}^{b} A_{\nu}^{c}
$$

and where

$$
L \equiv \log \left(\mu^{2} / p^{2}\right)
$$

with $p$ being an external momentum, and $\mu$ being a renormalization scale parameter. We find analogous to eq. (2.6) that the effective action is invariant under changes in the unphysical parameter $\mu$ :

$$
\begin{gathered}
0=\mu^{2} \frac{d \Gamma}{d \mu^{2}}=\left(\mu^{2} \frac{\partial}{\partial \mu^{2}}+\beta\left(g^{2}\right) \frac{\partial}{\partial g^{2}}+\frac{\gamma\left(g^{2}\right)}{2} \int d^{4} x A_{\eta}^{a}(x) \frac{\delta}{\delta A_{\eta}^{a}(x)}\right) \Gamma[A] \\
\beta\left(g^{2}\right)=\sum_{k=2}^{\infty} g^{2 k} b_{k} \equiv \mu^{2} \frac{d g^{2}(\mu)}{d \mu^{2}} \\
\frac{\gamma\left(g^{2}\right)}{2} A_{\eta}^{a}=\mu^{2} \frac{\partial A_{\eta}^{a}}{\partial \mu^{2}}(x, \mu) .
\end{gathered}
$$

Two features of Yang-Mills theory make the effective-action (4.1) more tractable than that of the $\phi_{6}^{3}$ model considered in Section 2. First of all, $g A_{\eta}^{a}$ is unrenormalized in order to preserve gauge invariance in the background field if gauge fixing is chosen appropriately [18, 19], in which case

$$
0=\mu^{2} \frac{d}{d \mu^{2}}\left[g(\mu) A_{\eta}^{a}(x, \mu)\right]=\left(\frac{\beta\left(g^{2}\right)}{2 g}\right) A_{\eta}^{a}(x, \mu)+g\left(\frac{\gamma\left(g^{2}\right)}{2} A_{\eta}^{a}(x, \mu)\right) .
$$

Consequently we find that

$$
\gamma\left(g^{2}\right)=-\beta\left(g^{2}\right) / g^{2}
$$

Secondly, we note that the logarithm $L$ in the effective action (4.1) has only explicit dependence on the renormalization mass scale $\mu^{2}$; by contrast the logarithm appearing in the effective action (2.2) has both explicit and implicit (through $m(\mu)$ ) dependence on $\mu^{2}$.

Upon substitution of eqs. (4.1) and (4.8) into the RG-equation (4.4), we obtain

$$
\left[\frac{\partial}{\partial L}+\beta\left(g^{2}\right)\left(\frac{\partial}{\partial g^{2}}-\frac{1}{g^{2}}\right)\right] S\left[L, g^{2}\right]=0 .
$$

As before, we can expand the effective action's scalar function as a double summation in $L$ and $g^{2}$

$$
S\left[L, g^{2}\right]=\sum_{n=0}^{\infty} \sum_{m=0}^{n} y_{n, m} g^{2 n} L^{m} .
$$

Upon substitution of this series and the $\beta$-function series (4.5) into the RG-equation (4.9), we find that the aggregate coefficient of $g^{2 p} L^{p-k}$ necessarily vanishes (for positive integer values of $p$ and $k$ with $k<p$ ):

$$
(p-k+1) y_{p, p-k+1}+\sum_{\ell=1}^{k}(p-\ell-1) b_{\ell+1} y_{p-\ell, p-k}=0 .
$$


The double summation (4.10) can, as before, be rearranged into the form

$$
S\left[L, g^{2}\right]=\sum_{n=0}^{\infty} g^{2 n} U_{n}\left(g^{2} L\right)
$$

where $\left(v=g^{2} L\right)$

$$
U_{n}(v) \equiv \sum_{k=n}^{\infty} y_{k, k-n} v^{k-n}
$$

The recursion relations (4.11) can be used to obtain an explicit set of first order linear differential equations for $U_{n}(v)$. If one multiplies (4.11) by $v^{p-k}$ and then sums over $p$ from $p=k$ to infinity $(k \geq 1)$, one finds that

$$
\begin{aligned}
0 & =\sum_{p=k}^{\infty}(p-(k-1)) y_{p, p-(k-1)} v^{p-k} \\
& +\sum_{\ell=1}^{k} b_{\ell+1}\left(v \sum_{p=k}^{\infty}(p-k) y_{p-\ell, p-k} v^{p-k-1}+(k-\ell-1) \sum_{p=k}^{\infty} y_{p-\ell, p-k} v^{p-k}\right) \\
& =\frac{d U_{k-1}}{d v}+\sum_{\ell=1}^{k} b_{\ell+1}\left(v \frac{d U_{k-\ell}}{d v}+(k-\ell-1) U_{k-\ell}\right) .
\end{aligned}
$$

Given the initial conditions

$$
U_{n}(0)=y_{n, 0}
$$

one may solve the differential equations (4.14) successively for $U_{0}, U_{1}, U_{2}$ etc. For example, the solutions to these equations accessible from the set of known coefficients $\left\{b_{2}, b_{3}, b_{4}, b_{5}\right\}$ of the Yang-Mills $\beta$-function are

$$
\begin{gathered}
U_{0}(v)=y_{0,0}\left(1+b_{2} v\right) \\
U_{1}(v)=y_{1,0}+\frac{b_{3}}{b_{2}} y_{0,0} \log \left(1+b_{2} v\right) \\
U_{2}(v)=\frac{y_{2,0}+y_{0,0}\left[\frac{b_{3}^{2}}{b_{2}^{2}} \log \left(1+b_{2} v\right)+\left(b_{4}-\frac{b_{3}^{2}}{b_{2}}\right) v\right]}{\left(1+b_{2} v\right)} \\
U_{3}(v)=\left[y_{3,0}-y_{2,0} \frac{b_{3}}{b_{2}} \log \left(1+b_{2} v\right)\right] /\left(1+b_{2} v\right)^{2} \\
+y_{0,0}\left\{\left[\frac{b_{5}}{2 b_{2}}-\frac{b_{3} b_{4}}{b_{2}^{2}}+\frac{b_{3}^{3}}{2 b_{2}^{3}}\right]+\left[\frac{b_{3} b_{4}}{b_{2}^{2}}-\frac{b_{3}^{3}}{b_{2}^{3}}\right] /\left(1+b_{2} v\right)\right. \\
\left.+\frac{\left[\frac{b_{3}^{3}}{2 b_{2}^{3}}-\frac{b_{5}}{2 b_{2}}+\frac{b_{3} b_{4}}{b_{2}^{2}} \log \left(1+b_{2} v\right)-\frac{b_{3}^{3}}{2 b_{2}^{3}} \log ^{2}\left(1+b_{2} v\right)\right]}{\left(1+b_{2} v\right)^{2}}\right\}
\end{gathered}
$$

where the $\overline{M S} S U(N)$ Yang-Mills $\beta$-function coefficients, as defined by eq. (4.5) are 20]

$$
\begin{gathered}
b_{2}=-\frac{11 N}{12\left(4 \pi^{2}\right)}, \quad b_{3}=-\frac{17 N^{2}}{24\left(4 \pi^{2}\right)^{2}}, \quad b_{4}=-\frac{2857 N^{3}}{3456\left(4 \pi^{2}\right)^{3}}, \\
b_{5}=-\frac{\left(N^{4}\left[\frac{150473}{486}+\frac{44}{9} \zeta(3)\right]+N^{2}\left[-\frac{40}{3}+352 \zeta(3)\right]\right)}{256\left(4 \pi^{2}\right)^{4}} .
\end{gathered}
$$


The utility of the solution (4.12) is limited, however, by our ignorance of the coefficients $y_{k, 0}$ appearing in eqs. (4.16)-(4.19). It is possible to achieve deeper insight into the behaviour of $S\left[L, g^{2}\right]$ using the methods of Section 3. As before, we expand the effective Lagrangian (4.1) in powers of $L$ :

$$
S\left[L, g^{2}\right] \equiv \sum_{n=0}^{\infty} B_{n}\left(g^{2}\right) L^{n}
$$

If we substitute this series into the RG equation (4.9) we find that

$$
(n+1) B_{n+1}+g^{2} \beta\left(g^{2}\right) \frac{d}{d g^{2}}\left(\frac{B_{n}\left(g^{2}\right)}{g^{2}}\right)=0
$$

Let $\tilde{B}_{n}\left(g^{2}\right) \equiv B_{n}\left(g^{2}\right) / g^{2}$, in which case

$$
\tilde{B}_{n+1}\left(g^{2}\right)=-\frac{\beta\left(g^{2}\right)}{(n+1)} \frac{d}{d g^{2}} \tilde{B}_{n}\left(g^{2}\right) .
$$

If we define a variable $y$ implicitly via the relation

$$
\beta\left(g^{2}\right) \frac{d}{d g^{2}} \equiv \frac{d}{d y}
$$

we see from eq. (4.23) that

$$
\tilde{B}_{n+1}(y)=-\frac{1}{(n+1)} \frac{d}{d y} \tilde{B}_{n}(y)
$$

in which case

$$
\begin{aligned}
S\left[L, g^{2}\right] & =g^{2}(\mu) \sum_{n=0}^{\infty} \tilde{B}_{n} L^{n} \\
& =g^{2}(\mu) \sum_{n=0}^{\infty}\left(-\frac{1}{n !} L^{n} \frac{d^{n}}{d y^{n}}\right) \tilde{B}_{0}\left(g^{2}[y]\right) \\
& =g^{2}(\mu) \exp \left[-L \frac{d}{d y}\right] \tilde{B}_{0}\left(g^{2}[y]\right) \\
& =g^{2}(\mu) \tilde{B}_{0}\left(g^{2}[y-L]\right) .
\end{aligned}
$$

Now the definition (4.24) implies that

$$
y\left[g^{2}(\mu)\right]=\int_{g^{2}(p)}^{g^{2}(\mu)} \frac{d \xi}{\beta(\xi)} .
$$

Note from eq. (4.27) that the implicit function $y$ has been chosen such that $g^{2}[y]=g^{2}(\mu)$, $g^{2}[0]=g^{2}(p)$; i.e. $y\left[g^{2}(p)\right]=0$. However, the defining relation (4.5) for the $\beta$-function necessarily implies that

$$
L \equiv \log \left(\frac{\mu^{2}}{p^{2}}\right)=\int_{g^{2}(p)}^{g^{2}(\mu)} \frac{d s}{\beta(s)} .
$$

Consequently, we see from eqs. (4.27) and (4.28) that $y=L$, in which case we find from eq. (4.26) that

$$
\begin{aligned}
S\left[L, g^{2}\right] & =g^{2}(\mu) \tilde{B}_{0}\left[g^{2}[0]\right] \\
& =g^{2}(\mu) \tilde{B}_{0}\left[g^{2}(p)\right] .
\end{aligned}
$$


Since $g^{2} \tilde{B}_{0}\left(g^{2}\right)=B_{0}\left(g^{2}\right)=\sum_{n=0}^{\infty} y_{n, 0} g^{2 n}$, we find that

$$
S\left[L, g^{2}\right]=\left(\sum_{n=0}^{\infty} y_{n, 0}[g(p)]^{2(n-1)}\right) g^{2}(\mu) .
$$

We thus see from eqs. (4.1) and (4.30) that the effective Lagrangian is proportional to $g^{2}(\mu) F_{\mu \nu}^{a} F^{\mu \nu a}$. Note from eq. (4.30) that $S=k g^{2}(\mu)$, where $k$ is independent of $\mu$, and that such a result is a valid solution to the RG-equation (4.9).2

The solution (4.30) is particularly useful in theories in which the $\beta$-function is known to all orders. Both the form (4.1) for the logarithm-dependent portion of the bosonic contribution to the effective Lagrangian and the RG-equation (4.9) remain applicable to $N=1$ supersymmetric Yang-Mills theory. For this supersymmetric case, however, the $\beta$-function can be obtained to all orders by requiring that the supermultiplet structure of the theory uphold the Adler-Bardeen theorem [15]. Indeed, the $\beta$-function obtained by this method is found to agree with that extracted by instanton calculus methods [14]; for the $S U(3)$ case this $\beta$-function is found to be

$$
\beta\left(g^{2}\right)=\mu^{2} \frac{d g^{2}}{d \mu^{2}}=-\frac{9 g^{4} / 16 \pi^{2}}{1-3 g^{2} / 8 \pi^{2}},
$$

in which case the couplant $x(\mu) \equiv g^{2}(\mu) / 16 \pi^{2}$ satisfies the constraint

$$
-\frac{1}{6 x(\mu)} \exp \left[-\frac{1}{6 x(\mu)}\right]=-\frac{1}{6 x(p)}\left(\frac{\mu^{2}}{p^{2}}\right)^{1 / 6} \exp \left[-\frac{1}{6 x(p)}\right] \text {. }
$$

Given the defining relationship $f e^{f}=\xi$ for the Lambert W-function $f=W[\xi]$, we obtain from eq. (4.32) the following closed-form expression for the $F^{2}$-dependent portion of the effective Lagrangian (4.30) of supersymmetric $N_{c}=3$ Yang-Mills theory:

$$
\Gamma[A]=\frac{2 \pi^{2}\left(\sum_{n=0}^{\infty} y_{n, 0}[g(p)]^{2(n-1)}\right) F_{\mu \nu}^{a}(A) F^{\mu \nu a}(A)}{3 W\left[-\frac{8 \pi^{2}}{3 g^{2}(p)}\left(\frac{\mu^{2}}{p^{2}}\right)^{1 / 6} \exp \left(-\frac{-8 \pi^{2}}{3 g^{2}(p)}\right)\right]}
$$

\footnotetext{
${ }^{2}$ The result $S=k g^{2}(\mu)$ can be extracted trivially from eqs. (4.1) and (4.2) by noting that the scale invariance of $g(\mu) A_{\nu}^{a}(x, \mu)$ [eq. (4.7)], implies concomitant scale invariance of $g(\mu) F_{\mu \nu}^{a}$, and consequently, of $g^{2}(\mu) F_{\mu \nu}^{a} F^{\mu \nu a}$.
} 


\section{The RG-Invariant Effective Couplant}

An unanticipated consequence of the previous section is a new and useful series representation for the RG-invariant couplant $x(p) \equiv g^{2}(p) / 4 \pi^{2}$ (for QCD $x(\sqrt{s})=\alpha_{s}(\sqrt{s}) / 4 \pi$ ) that does not involve an order-by-order redefinition of the scale parameter $\Lambda$. To obtain this series, we first note that eqs. (4.16)-(4.19) and eq. (4.30) are derived for arbitrary values of $y_{n, 0}$. We further note that if one were to choose $y_{n, 0}=\delta_{n, 0}$, then the expression (4.30) would just be $g^{2}(\mu) / g^{2}(p)$. However, if we make the choice $y_{n, 0}=\delta_{n, 0}$ in eqs. (4.16)-(4.19), the series expansion (4.12) corresponding to the same quantity as eq. (4.30) is seen to be

$$
\begin{aligned}
\frac{g^{2}(\mu)}{g^{2}(p)} & =w+g^{2}(\mu) \frac{b_{3}}{b_{2}} \log (w) \\
& +\frac{g^{4}(\mu)}{w}\left[\frac{b_{3}^{2}}{b_{2}^{2}} \log (w)+\left(\frac{b_{4}}{b_{2}}-\frac{b_{3}^{2}}{b_{2}^{2}}\right)(w-1)\right] \\
& +\frac{g^{6}(\mu)}{w^{2}}\left[\left(\frac{b_{5}}{2 b_{2}}-\frac{b_{3} b_{4}}{b_{2}^{2}}+\frac{b_{3}^{3}}{2 b_{2}^{3}}\right) w^{2}\right. \\
& +\left(\frac{b_{3} b_{4}}{b_{2}^{2}}-\frac{b_{3}^{3}}{b_{2}^{3}}\right) w+\left(\frac{b_{3}^{3}}{2 b_{2}^{3}}-\frac{b_{5}}{2 b_{2}}\right) \\
& \left.+\frac{b_{3} b_{4}}{b_{2}^{2}} \log (w)-\frac{b_{3}^{3}}{2 b_{2}^{3}} \log ^{2}(w)\right]+\mathcal{O}\left[g^{8}(\mu)\right]
\end{aligned}
$$

where

$$
w \equiv 1+b_{2} g^{2}(\mu) \log \left(\frac{\mu^{2}}{p^{2}}\right),
$$

and where the constants $b_{k}$ are as defined by the $\beta$-function (4.5). For our purposes here, we will utilize the (somewhat more) standard conventions of QCD:

$$
\begin{gathered}
x(\mu)=\alpha_{s}(\mu) / \pi=g^{2}(\mu) / 4 \pi^{2} \\
\mu^{2} \frac{d}{d \mu^{2}} x(\mu)=-\sum_{k=0}^{\infty} \beta_{k}(x(\mu))^{k+2} .
\end{gathered}
$$

Comparing eqs. (4.5) and (5.4), we see that

$$
b_{k+2}=-\beta_{k} /\left(4 \pi^{2}\right)^{k+1}
$$

and that

$$
w=1-\beta_{0} x(\mu) \log \left(\mu^{2} / p^{2}\right) .
$$

Using the definition (5.3) and the $\beta$-function coefficients defined by eqs. (5.4) and (5.5), we find from eq. (5.1) that

$$
\begin{aligned}
\frac{x(\mu)}{x(p)} & =w+x(\mu) \frac{\beta_{1}}{\beta_{0}} \log (w) \\
& +\frac{x^{2}(\mu)}{w}\left[\frac{\beta_{1}^{2}}{\beta_{0}^{2}} \log (w)+\left(\frac{\beta_{2}}{\beta_{0}}-\frac{\beta_{1}^{2}}{\beta_{0}^{2}}\right)(w-1)\right] \\
& +\frac{x^{3}(\mu)}{w^{2}}\left[\left(\frac{\beta_{3}}{2 \beta_{0}}-\frac{\beta_{1} \beta_{2}}{\beta_{0}^{2}}+\frac{\beta_{1}^{3}}{2 \beta_{0}^{3}}\right) w^{2}\right. \\
& +\left(\frac{\beta_{1} \beta_{2}}{\beta_{0}^{2}}-\frac{\beta_{1}^{3}}{\beta_{0}^{3}}\right) w+\left(\frac{\beta_{1}^{3}}{2 \beta_{0}^{3}}-\frac{\beta_{3}}{2 \beta_{0}}\right) \\
& \left.+\frac{\beta_{1} \beta_{2}}{\beta_{0}^{2}} \log (w)-\frac{\beta_{1}^{3}}{2 \beta_{0}^{3}} \log ^{2}(w)\right] \\
& +\mathcal{O}\left(x^{4}(\mu)\right),
\end{aligned}
$$


with $w$ given by eq. (5.6).

Now the expression we seek to obtain is an expression for the RG-invariant couplant $x(p)$, where $p$ is an external physical momentum scale, in terms of a chosen reference momentum scale $\mu$ and a reference couplant value $x(\mu)$. As an obvious example, the solution of eq. (5.4) when only the leading one-loop (1L) coefficient $\beta_{0}$ contributes to the right-hand side is just

$$
x_{1 L}(p)=\frac{x(\mu)}{1-\beta_{0} x(\mu) \log \left(\frac{\mu^{2}}{p^{2}}\right)}=\frac{x(\mu)}{w}
$$

For the full $\beta$-function (5.4), our solution must necessarily be of the form

$$
x(p)=x(\mu)\left[S_{0}+x(\mu) S_{1}+x^{2}(\mu) S_{2}+x^{3}(\mu) S_{3}+\ldots\right],
$$

where the coefficients $S_{0}, S_{1}, S_{2}, S_{3}$ are functions of $x(\mu)$ and $w$. To find these coefficients algebraically, we simply require that the product of $x(\mu) / x(p)$, as given by eq. (5.7), and $x(p) / x(\mu)$ as given by eq. (5.9), be equal to unity on an order-by-order basis in $x(\mu)$ :

$$
\begin{gathered}
w S_{0}=1 \\
w S_{1}+S_{0} \frac{\beta_{1}}{\beta_{0}} \log (w)=0 \\
w S_{2}+S_{1} \frac{\beta_{1}}{\beta_{0}} \log (w)+\frac{S_{0}}{w}\left[\frac{\beta_{1}^{2}}{\beta_{0}^{2}} \log (w)+\left(\frac{\beta_{2}}{\beta_{0}}-\frac{\beta_{1}^{2}}{\beta_{0}^{2}}\right)(w-1)\right]=0 \\
w S_{3}+S_{2} \frac{\beta_{1}}{\beta_{0}} \log (w)+\frac{S_{1}}{w}\left[\frac{\beta_{1}^{2}}{\beta_{0}^{2}} \log (w)+\left(\frac{\beta_{2}}{\beta_{0}}-\frac{\beta_{1}^{2}}{\beta_{0}^{2}}\right)(w-1)\right] \\
+\frac{S_{0}}{w^{2}}\left[\left(\frac{\beta_{3}}{2 \beta_{0}}-\frac{\beta_{1} \beta_{2}}{\beta_{0}^{2}}+\frac{\beta_{1}^{3}}{2 \beta_{0}^{3}}\right) w^{2}+\left(\frac{\beta_{1} \beta_{2}}{\beta_{0}^{2}}-\frac{\beta_{1}^{3}}{\beta_{0}^{3}}\right) w\right. \\
\left.+\left(\frac{\beta_{1}^{3}}{2 \beta_{0}^{3}}-\frac{\beta_{3}}{2 \beta_{0}}\right)+\frac{\beta_{1} \beta_{2}}{\beta_{0}^{2}} \log (w)-\frac{\beta_{1}^{3}}{2 \beta_{0}^{3}} \log ^{2}(w)\right]=0 .
\end{gathered}
$$

By solving these equations sequentially, and the substituting into eq. (5.9), we find that

$$
\begin{aligned}
x(p) & =\frac{x(\mu)}{w}-\frac{x^{2}(\mu)}{w^{2}} \frac{\beta_{1}}{\beta_{0}} \log (w) \\
& +\frac{x^{3}(\mu)}{w^{3}}\left[\left(\frac{\beta_{1}^{2}}{\beta_{0}^{2}}-\frac{\beta_{2}}{\beta_{0}}\right)(w-1)-\frac{\beta_{1}^{2}}{\beta_{0}^{2}}\left(\log (w)-\log ^{2}(w)\right)\right] \\
& +\frac{x^{4}(\mu)}{w^{4}}\left[\left(-\frac{\beta_{3}}{2 \beta_{0}}+\frac{\beta_{1} \beta_{2}}{\beta_{0}^{2}}-\frac{\beta_{1}^{3}}{2 \beta_{0}^{3}}\right) w^{2}+\left(\frac{\beta_{1}^{3}}{\beta_{0}^{3}}-\frac{\beta_{1} \beta_{2}}{\beta_{0}^{2}}\right) w\right. \\
& +\left(\frac{2 \beta_{1} \beta_{2}}{\beta_{0}^{2}}-\frac{2 \beta_{1}^{3}}{\beta_{0}^{3}}\right) w \log (w) \\
& +\left(\frac{\beta_{3}}{2 \beta_{0}}-\frac{\beta_{1}^{3}}{2 \beta_{0}^{3}}\right)+\left(\frac{2 \beta_{1}^{3}}{\beta_{0}^{3}}-\frac{3 \beta_{1} \beta_{2}}{\beta_{0}^{2}}\right) \log (w) \\
& \left.+\frac{\beta_{1}^{3}}{\beta_{0}^{3}}\left(\frac{5}{2} \log ^{2}(w)-\log ^{3}(w)\right)\right]+\mathcal{O}\left(x^{5}(\mu)\right) .
\end{aligned}
$$

The leading three orders of the above equation have been derived previously (see eq. (16) of ref. [12]). In Appendix B, we derive eq. (5.14) via an alternative procedure utilizing the explicit RG-invariance of the couplant $x(p)$. This expression may be interpreted as an expression for 
the four-loop effective couplant in terms of $x(\mu)$ and the one-loop couplant $x_{1 L}(p)$, as given by eq. (5.8):

$$
\begin{aligned}
x_{4 L}(p) & =x_{1 L}(p)-\frac{\beta_{1}}{\beta_{0}} x_{1 L}^{2}(p) \log \left(\frac{x(\mu)}{x_{1 L}(p)}\right) \\
& +x_{1 L}^{3}(p)\left[\left(\frac{\beta_{1}^{2}}{\beta_{0}^{2}}-\frac{\beta_{2}}{\beta_{0}}\right)\left(\frac{x(\mu)}{x_{1 L}(p)}-1\right)\right. \\
& -\frac{\beta_{1}^{2}}{\beta_{0}^{2}}\left(\log \left(\frac{x(\mu)}{x_{1 L}(p)}\right)-\log ^{2}\left(\frac{x(\mu)}{x_{1 L}(p)}\right)\right] \\
& +x_{1 L}^{4}(p)\left[\left(-\frac{\beta_{3}}{2 \beta_{0}}+\frac{\beta_{1} \beta_{2}}{\beta_{0}^{2}}-\frac{\beta_{1}^{3}}{2 \beta_{0}^{3}}\right)\left(\frac{x(\mu)}{x_{1 L}(p)}\right)^{2}\right. \\
& +\left(\frac{\beta_{1}^{3}}{\beta_{0}^{3}}-\frac{\beta_{1} \beta_{2}}{\beta_{0}^{2}}\right)\left(\frac{x(\mu)}{x_{1 L}(p)}\right) \\
& +\left(\frac{2 \beta_{1} \beta_{2}}{\beta_{0}^{2}}-\frac{2 \beta_{1}^{3}}{\beta_{0}^{3}}\right)\left(\frac{x(\mu)}{x_{1 L}(p)}\right) \log \left(\frac{x(\mu)}{x_{1 L}(p)}\right) \\
& +\left(\frac{\beta_{3}}{2 \beta_{0}}-\frac{\beta_{1}^{3}}{2 \beta_{0}^{3}}\right)+\left(\frac{2 \beta_{1}^{3}}{\beta_{0}^{3}}-\frac{3 \beta_{1} \beta_{2}}{\beta_{0}^{2}}\right) \log \left(\frac{x(\mu)}{x_{1 L}(p)}\right) \\
& \left.+\frac{\beta_{1}^{3}}{\beta_{0}^{3}}\left(\frac{5}{2} \log ^{2}\left(\frac{x(\mu)}{x_{1 L}(p)}\right)-\log ^{3}\left(\frac{x(\mu)}{x_{1 L}(p)}\right)\right)\right] \\
& +\mathcal{O}\left(x_{1 L}^{5}(p)\right) .
\end{aligned}
$$

In Fig. 1, we display for $n_{f}=3$ QCD a comparison of the running couplant (5.14) to the corresponding explicit (numerical) solution to the differential equation (5.4) truncated after the last known contribution $\left(-\beta_{3} x^{5}\right)$. To obtain these plots, we assume that $\mu=m_{\tau}$ and that $x\left(m_{\tau}\right)=\alpha_{s}\left(m_{\tau}\right) / \pi=0.33 / \pi$ [21]. The two curves are seen to coincide until $\sqrt{p^{2}}<1$ $\mathrm{GeV}$, in which case the expression (5.14) falls below the true couplant value. This discrepancy follows from the fact that the series (5.14) is term-by-term singular at $w=0$, corresponding to the Landau pole of the one-loop couplant (5.8). However, the Landau pole of the true 4loop couplant, as evolved directly from the differential equation (5.4), is necessarily above the $w=0$ one-loop pole at $p^{2}=m_{\tau}^{2} \exp \left[-1 / \beta_{0} x\left(m_{\tau}\right)\right]$ because $\left\{\beta_{0}, \beta_{1}, \beta_{2}, \beta_{3}\right\}$ are all positive; i.e. the four-loop $\beta$-function drives the couplant more quickly to large values than the one-loop $\beta$-function.

This behaviour becomes somewhat more transparent if we specialize to $n_{f}=3$ QCD in the 't Hooft renormalization scheme [22] in which $\beta_{0}$ and $\beta_{1}$ have their $\overline{M S}$ values, but all subsequent $\beta$-function coefficients are zero. In Fig. 2 we display successively higher order plots of the series (5.9) in the 't Hooft scheme with the same initial conditions at $m_{\tau}$ as in Fig. 1. The figure displays the lowest-order $(1 \mathrm{~L})$ approximation $x(p) \cong x(\mu) S_{0}$, the next-to-lowest (NL) order approximation $x(p) \cong x(\mu)\left(S_{0}+x(\mu) S_{1}\right)$, the subsequent (NNL) approximation $x(p) \cong x(\mu)\left(S_{0}+x(\mu) S_{1}+x^{2}(\mu) S_{2}\right)$, and finally the NNNL approximation $x(p) \cong x(\mu)\left(S_{0}+x(\mu) S_{1}+x^{2}(\mu) S_{2}+x^{3}(\mu) S_{3}\right)$. The functions $S_{0}$ and $S_{1}$ are as given in eq. (5.14) [or alternatively, eqs. (B.16) and (B.19) of Appendix B]. The functions $S_{2}$ and $S_{3}$ are also given in eq. (5.14) [or eqs. (B.22) and (B.23)], but with $\beta_{2}$ and $\beta_{3}$ in these equations taken to be zero, consistent with the 't Hooft renormalization scheme. Since the lowest order approximation is just the one-loop running couplant (5.8), the figure shows how the incorporation of successive terms in the series (5.9) takes one from the one-loop couplant $x_{1 L}(p)$ to approximations that grow successively closer to the true 't Hooft scheme ('tH) couplant, defined as the solution of eq. (5.4) with $\beta_{k}=0$ for $k \geq 2$.

However, it is also evident from the figure that all the series approximations listed above 
differ from the true solution in the infrared region. Each approximation to the series (5.9) is term-by-term singular at $w=0$, the Landau pole of $x_{1 L}(p)$ [eq. (5.8)] which occurs (for $\mu=m_{\tau}$ ) at

$$
p^{2}=m_{\tau}^{2} \exp \left[-1 / \beta_{0} x\left(m_{\tau}\right)\right] .
$$

By contrast, the exact 't Hooft scheme solution to eq. (5.4) satisfies the constraint

$$
\begin{aligned}
\beta_{0} \log \left(\frac{m_{\tau}^{2}}{p^{2}}\right) & =\frac{1}{x\left(m_{\tau}\right)}-\frac{1}{x(p)} \\
& +\frac{\beta_{1}}{\beta_{0}} \log \left[\frac{x\left(m_{\tau}\right)\left[x(p)+\beta_{0} / \beta_{1}\right]}{x(p)\left[x\left(m_{\tau}\right)+\beta_{0} / \beta_{1}\right]}\right] .
\end{aligned}
$$

The Landau pole of eq. (5.17) occurs when $x(p) \rightarrow \infty$, i.e., when

$$
p^{2}=m_{\tau}^{2} \exp \left[-1 / \beta_{0} x\left(m_{\tau}\right)\right]\left[1+\frac{\beta_{0}}{\beta_{1} x\left(m_{\tau}\right)}\right]^{\frac{\beta_{1}}{\beta_{0}^{2}}} .
$$

Since $\beta_{0}$ and $\beta_{1}$ are both positive, the true 't Hooft-scheme Landau pole (5.18) is clearly larger than the singularity (5.16) characterizing each term of the series (5.9). Thus, the true 't Hooft scheme couplant necessarily evolves faster than any approximation based upon the series (5.9).

Indeed, the true couplant has an infrared bound (5.18) on its domain that is well above the corresponding bound (5.16) on the terms of the series (5.9). Such a discrepancy between a series representation of a function and the function itself is not unprecedented. An illustrative toy example is the function

$$
f(s)=\left(s-\Lambda^{2}\right) /\left(s-\Lambda^{2}+x(s)\right),
$$

which is singular only at values of $s$ satisfying the constraint $s=\Lambda^{2}-x(s)$. A perturbative expansion of this same function in the expansion parameter $x(s)$,

$$
f(s)=1-\frac{x(s)}{s-\Lambda^{2}}+\frac{x^{2}(s)}{\left(s-\Lambda^{2}\right)^{2}}-\frac{x^{3}(s)}{\left(s-\Lambda^{2}\right)^{3}} \pm \ldots
$$

is term-by-term singular at $s=\Lambda^{2}$, as well as at any "Landau pole" values of $s$ at which $x(s)$ is itself singular. The analogy to calculable perturbative processes is inescapable; the occurrence of term-by-term singularities at some value of $s$ in a perturbative field- theoretical series for a physical process does not necessarily imply that the process itself is inaccessible at that value of $s$. Restrictions, such as a Landau pole, on the kinematical domain of the running couplant, do not necessarily correspond to infrared restrictions on the kinematical domain of the process itself.

\section{Instanton Contribution to the $S U(2)$ Effective Lagrangian}

Consider the instanton contribution to the effective Lagrangian of an $S U(2)$ gauge theory, as derived by 't Hooft [16]. This contribution contains an integral over the instanton size $\rho$

$$
\mathcal{L}_{\text {eff }} \sim K \int d \rho \rho^{-5+3 n_{f}} \exp \left\{-\frac{8 \pi^{2}}{g^{2}(\mu)} S\right\}
$$

where the series $S$ is a power series in the scale dependent coupling constant of the form

$$
S=1+\sum_{n=1}^{\infty} \sum_{m=0}^{n} T_{n, m} g^{2 n}(\mu) \log ^{m}(\mu \rho) .
$$


The contribution to the measure $K$ in eq. (6.1) is independent of the renormalization scale parameter $\mu$. In ref. [16], the one loop contributions $T_{1,0}$ and $T_{1,1}$ to eq. (6.2) are utilized explicitly to show that the integral (6.1) converges in the ultraviolet limit $(\rho \rightarrow 0)$ but diverges in the infrared limit $(\rho \rightarrow \infty)$. In this section, we explore whether the summation of successively subleading logarithms affects such asymptotic behaviour.

We begin by regrouping the series (6.2) in terms of sequential summations of leading $\left(S_{0}\right)$, next-to-leading $\left(S_{1}\right)$, next-to-next-to-leading $\left(S_{2}\right), \ldots$, logarithmic terms

$$
\begin{aligned}
S & =\sum_{k=0}^{\infty} g^{2 k}(\mu)\left(\sum_{\ell=k}^{\infty} T_{\ell, \ell-k}\left[g^{2}(\mu) \log (\mu \rho)\right]^{\ell-k}\right) \\
& \equiv \sum_{k=0}^{\infty} g^{2 k}(\mu) S_{k}\left[g^{2}(\mu) \log (\mu \rho)\right] .
\end{aligned}
$$

The RG-equation for the argument of the exponent occurring in the integrand of eq. (6.1) is just

$$
0=\left(\mu \frac{\partial}{\partial \mu}+\beta(g) \frac{\partial}{\partial g}\right) \sum_{k=0}^{\infty}[g(\mu)]^{2(k-1)} S_{k}\left[g^{2}(\mu) \log (\mu \rho)\right],
$$

where $\beta(g)$ is the $S U(2) \beta$-function with fermionic and scalar field (s.f.) contributions:

$$
\begin{gathered}
\mu \frac{d g}{d \mu}=\beta(g)=\sum_{k=1}^{\infty} b_{2 k+1} g^{2 k+1} \\
b_{3}=-\left(\frac{22}{3}-\frac{2 n_{f}}{3}-\text { s.f. }\right) / 16 \pi^{2} .
\end{gathered}
$$

If one employs the series definition (6.3) for the $S_{k}$ 's occurring on the right hand side of (6.4), one can obtain recursion relations for the $T_{n, m}$ coefficients. One finds from $S_{0}$, for example, that the aggregate coefficient of $g^{2(n-1)} L^{n-1}$ on the right hand side of (6.4) will vanish only if

$$
n T_{n, n}+b_{3}(2 n-4) T_{n-1, n-1}=0 .
$$

This recursion relation not only implies that $T_{1,1}=2 b_{3}$, a result explicitly obtained in ref. [16], 3 but also implies that $T_{n, n}=0$ for $n \geq 2$. Consequently, the summation of leading logarithm terms truncates at one-loop order and fails to alter the asymptotics already obtained; i.e.

$$
S_{0}\left[g^{2}(\mu) \log (\mu \rho)\right]=1+2 b_{3} g^{2}(\mu) \log (\mu \rho) \equiv w .
$$

For summations of subsequent subleading logarithms, one may rewrite the RG-equation (6.4) as

$$
0=\sum_{k=0}^{\infty} g^{2 k}\left[\frac{d S_{k}}{d u}+\sum_{\ell=0}^{k} b_{3+2(k-\ell)}\left[(2 \ell-2) S_{\ell}+2 u \frac{d S_{\ell}}{d u}\right]\right],
$$

where we have replaced $g^{2}(\mu) \log (\mu \rho)$ with the variable $u$. Since the right-hand side of $(6.9)$ is a power series in $g$ that must vanish order-by-order, we find after a little algebra that $S_{k}$ satisfies a first-order inhomogeneous linear differential equation in the variable $w=1+2 b_{3} u$,

$$
\frac{d S_{k}}{d w}+\frac{(k-1)}{w} S_{k}=-\frac{1}{2 b_{3} w}\left[\sum_{\ell=0}^{k-1} b_{3+2(k-\ell)}\left[2(\ell-1) S_{\ell}+2(w-1) \frac{d S_{\ell}}{d w}\right]\right], \quad(k \geq 1)
$$

\footnotetext{
${ }^{3}$ The RG-consistency of this result is also noted in ref. [16].
} 
with initial conditions following from the definition (6.3) $\left[u=g^{2}(\mu) \log (\mu \rho)\right]$

$$
\lim _{w \rightarrow 1} S_{k}=\lim _{u \rightarrow 0} S_{k}=T_{k, 0} .
$$

The solution to eq. (6.10) for $S_{k}[w]$ with this initial condition is given by

$$
\begin{aligned}
S_{k}[w] & =T_{k, 0} / w^{k-1} \\
& -\frac{1}{2 b_{3} w^{k-1}} \int_{1}^{w} d t t^{k-2}\left\{\sum_{\ell=0}^{k-1} b_{3+2(k-\ell)}\left[(2 \ell-2) S_{\ell}[t]+2(t-1) S_{\ell}^{\prime}[t]\right]\right\} .
\end{aligned}
$$

We see from eq. (6.8) that

$$
\begin{gathered}
S_{1}=T_{1,0}+\frac{b_{5}}{b_{3}} \log |w| \\
S_{2}=\left(\frac{b_{7}}{b_{3}}-\frac{b_{5}^{2}}{b_{3}^{2}}\right)+\frac{T_{2,0}-\left[\frac{b_{7}}{b_{3}}-\frac{b_{5}^{2}}{b_{3}^{2}}\right]+\frac{b_{5}^{2}}{b_{3}^{2}} \log |w|}{w} .
\end{gathered}
$$

Moreover, we find that the curly-bracketed contribution to the integrand of (6.12) arising from eqs. (6.8), (6.13) and (6.14) is of the form $\{\ldots\}=$ constant $+\mathcal{O}(1 / t)$, which ensures that $S_{3}[w]$ is of the same "constant $+\mathcal{O}(1 / w)$ " form as $S_{2}[w]$. Hence the $\ell=3$ contribution to the curlybracketed expression to (6.12), and by iteration all subsequent- $\ell$ contributions, will be of the form $\{\ldots\}=$ constant $+\mathcal{O}(1 / t)$, in which case we easily see from eq. (6.12) that in the large- $w$ limit

$$
S_{k}[w]=c_{k}+\mathcal{O}(1 / w), \quad k \geq 2 .
$$

Note that $c_{k}$ is a constant entirely obtained from $\beta$-function coefficients, as in eq. (6.14), and does not depend on the coefficients $T_{k, 0}$ calculated from field theory.

If we substitute the results (6.8), (6.13) and (6.15) into the series $S$ appearing in (6.1), we find that the $\rho$-dependence of the integrand of (6.1) in the $\rho \rightarrow 0$ or $\rho \rightarrow \infty$ limit is

$$
\begin{array}{r}
\rho^{-5+3 n_{f}} \exp \left[-\frac{8 \pi^{2}}{g^{2}} \sum_{k=0}^{\infty} g^{2 k} S_{k}\right] \\
\underset{|\log \rho| \rightarrow \infty}{\longrightarrow} \rho^{\frac{7}{3}\left(1+n_{f}\right)-s \cdot f \cdot|\log \rho|^{-8 \pi^{2} b_{5} / b_{3}} .}
\end{array}
$$

The exponent of $\rho$, which is obtained in ref. [16], follows via eq. (6.6) entirely from the leading-log $T_{1,1} g^{2} \log (\mu \rho)$ contribution. The exponent of $|\log \rho|$ follows from the summation (6.13) of nextto-leading logarithms; all subsequent summations (6.15) of logarithms generate contributions independent of $\rho$ in the large- $\rho$ limit. Thus, the result (6.16) is obtained via summation of all logarithms contributing to the series $S$, and is independent of any requirement that $g^{2}(\mu)$ be small. Surprisingly, we find only a very minimal amelioration of the infrared (large- $\rho$ ) divergent behaviour characterising the integral (6.1). Moreover, if the scalar field (s.f.) contribution were sufficiently large to render the exponent of $\rho$ in eq. (6.16) negative, the resulting ultraviolet divergence at $\rho=0$ would persist upon incorporating the summation of higher order logarithms.

\section{Acknowledgements}

We are grateful for discussions with V. A. Miransky, and for research support from the Natural Sciences and Engineering Research Council of Canada. R. and D. MacKenzie provided helpful suggestions. 


\section{Appendix A: $\mathcal{O}\left(\lambda^{2}\right)$-Equivalence of Two Expressions for $A(\lambda, L)$}

Two "lowest-order" RG-improved expressions for $A(\lambda, L)$ in the $\phi_{6}^{3}$ effective action (2.2) are

$$
\begin{gathered}
A(\lambda, L)=x^{D_{2} / B_{3}}, \\
A(\lambda, L)=\left[\frac{K W\left[\frac{2}{K} \exp \left(\frac{2 x}{K}\right)\right]}{2}\right]^{D_{2} / B_{3}},
\end{gathered}
$$

where

$$
x \equiv 1-B_{3} \lambda^{2} L, \quad K \equiv G_{2} \lambda^{2} .
$$

[Eqs.

(A.1) and (A.2) are eqs. (2.28) and (3.15) of the text, respectively.] In this appendix we will demonstrate that eqs. (A.1) and (A.2) differ by at most $\mathcal{O}\left(\lambda^{4}\right)$.

We first note that both expressions are equal to unity when $L=\log \left(\frac{m^{2}}{\mu^{2}}\right)=0$, hence, when $x=1$. To see this for the second expression, we note that the Lambert W-function in eq. (A.2) satisfies the constraint

$$
W\left[\frac{2}{K} e^{2 / K}\right] \exp \left(W\left[\frac{2}{K} e^{2 / K}\right]\right)=\frac{2}{K} \exp \left(\frac{2}{K}\right),
$$

in which case $W\left[\frac{2}{K} e^{2 / K}\right]=2 / K$, and $A(\lambda, L)$, as defined by eq. (A.2), is equal to 1 when $x=1$.

Suppose we now perturb eq. (A.2) about $K=0$. To compare eqs. (A.2) and (A.1) with $\lambda$ small, we utilise the following lowest order deviation:

$$
\begin{aligned}
\frac{K W\left[\frac{2}{K} \exp (2 x / K)\right]}{2} & =\lim _{K \rightarrow 0}\left(\frac{K W\left[\frac{2}{K} \exp [2 x / K]\right]}{2}\right) \\
& +K \lim _{K \rightarrow 0}\left[\frac{d}{d K}\left(\frac{K W\left[\frac{2}{K} \exp \left(\frac{2 x}{K}\right)\right]}{2}\right)\right] \\
& +\mathcal{O}\left(K^{2}\right) .
\end{aligned}
$$

The first limit on the right-hand side of eq. (A.5) is $x$, and the second limit is $-\log (x) / 2$, as we shall show below. Consequently, we have

$$
\begin{aligned}
\frac{K W\left[\frac{2}{K} \exp (2 x / K)\right]}{2} & =x-\frac{K}{2} \log (x)+\mathcal{O}\left(K^{2}\right) \\
& =x+\frac{G_{2} B_{3}}{2} \lambda^{4} L+\mathcal{O}\left(\lambda^{4}\right),
\end{aligned}
$$

as $K^{2}=G_{2}^{2} \lambda^{4}$ and $\log x=-B_{3} \lambda^{2} L+\mathcal{O}\left(\lambda^{4}\right)$. Upon substitution of eq. (A.6) into eq. (A.2), we find that eq. (A.2) deviates from eq. (A.1) at most by order $\lambda^{4}$, as stated at the end of Section 3 .

To complete this demonstration, we evaluate explicitly the limits on the right-hand side of eq. (A.5). Defining $\eta=2 / K$, we find via L'Hôpital's rule that

$$
\begin{aligned}
L_{1} & \equiv \lim _{K \rightarrow 0} \frac{K W\left[\frac{2}{K} \exp (2 x / K)\right]}{2}=\lim _{\eta \rightarrow \infty} \frac{W\left[\eta e^{\eta x}\right]}{\eta} \\
& =\lim _{\eta \rightarrow \infty} \frac{W^{\prime}\left[\eta e^{\eta x}\right](1+\eta x) e^{\eta x}}{1} .
\end{aligned}
$$


One can easily show from the defining relation $W(\xi) \exp [W(\xi)]=\xi$ that $W^{\prime}(\xi)=W(\xi) /[\xi(W(\xi)+1)]$, in which case

$$
\begin{aligned}
L_{1} & =\lim _{\eta \rightarrow \infty} \frac{W\left(\eta e^{\eta x}\right)(1+\eta x)}{\eta\left[1+W\left(\eta e^{\eta x}\right)\right]} \\
& =\left(\lim _{W \rightarrow \infty} \frac{W}{1+W}\right) x=x,
\end{aligned}
$$

justifying the first term on the right-hand side of eq. (A.6).

To evaluate the second limit on the right-hand side of eq. (A.6), we first note that

$$
\frac{d}{d K}\left(\frac{K W\left[\frac{2}{K} \exp \left(\frac{2 x}{K}\right)\right]}{2}\right)=\frac{W\left[\frac{2}{K} \exp \left(\frac{2 x}{K}\right)\right]\left[K W\left[\frac{2}{K} \exp \left(\frac{2 x}{K}\right)\right]-2 x\right]}{2 K\left[W\left[\frac{2}{K} \exp \left(\frac{2 x}{K}\right)\right]+1\right]}
$$

Since $\lim _{W \rightarrow \infty} W /(W+1)=1$, we find that

$$
\begin{aligned}
L_{2} & \equiv \lim _{K \rightarrow 0} \frac{d}{d K}\left(\frac{K W\left[\frac{2}{K} \exp \left(\frac{2 x}{K}\right)\right]}{2}\right)=\lim _{K \rightarrow 0}\left(\frac{K W\left[\frac{2}{K} \exp \left(\frac{2 x}{K}\right)\right]-2 x}{2 K}\right) \\
& =\lim _{\eta \rightarrow \infty} \frac{\left(W\left[\eta e^{\eta x}\right]-\eta x\right)}{2}
\end{aligned}
$$

We exponentiate eq. (A.10) and use the Lambert function relation $\exp [W(\xi)]=\xi / W(\xi)$ to find that

$$
\begin{aligned}
\exp \left(2 L_{2}\right) & =\lim _{\eta \rightarrow \infty} \exp \left[W\left[\eta e^{\eta x}\right]\right] e^{-\eta x} \\
& =\lim _{\eta \rightarrow \infty}\left(\frac{\eta}{W\left[\eta e^{\eta x}\right]}\right)=\frac{1}{x}
\end{aligned}
$$

The final step of eq. (A.11) follows from the evaluation of $L_{1}=\lim _{\eta \rightarrow \infty} \frac{W\left[\eta e^{\eta x}\right]}{\eta}=x$, as obtained from eqs. (A.7) and (A.8). Taking the natural logarithm of both sides of eq. (A.11), we find that

$$
L_{2}=-\frac{1}{2} \log (x)
$$

justifying the second term on the right-hand side of eq. (A.6). 


\section{Appendix B: Explicit Derivation of the RG-Resummed Ef- fective Couplant}

In this appendix we sum leading and three subsequent subleading orders of logarithmic terms within the series expansion

$$
x(p)=x(\mu) \sum_{n=0}^{\infty} \sum_{m=0}^{n} T_{n, m} x^{n}(\mu) \log ^{m}\left(\mu^{2} / p^{2}\right)
$$

for the running couplant, as defined by the differential equation

$$
\mu^{2} \frac{d x}{d \mu^{2}}(\mu)=\beta[x(\mu)],
$$

with $x(p)$ as an initial condition that is necessarily independent of the variable $\mu$. For example, if $\beta=-\beta_{0} x^{2}(\mu)$, one can solve eq. (B.2) directly to obtain the explicit one-loop (1L) result

$$
x_{1 L}(p)=\frac{x(\mu)}{1-\beta_{0} x(\mu) \log \left(\mu^{2} / p^{2}\right)} .
$$

When one goes beyond one loop order, however, the solution to eq. (B.2) is no longer explicit, but implicitly defined via the constraint

$$
\log \left(\frac{p^{2}}{\mu^{2}}\right)-\int_{x(\mu)}^{x(p)} \frac{d s}{\beta(s)}=0 .
$$

In this appendix, we will apply the RG-equation

$$
\mu^{2} \frac{d}{d \mu^{2}} x(p)=0
$$

to the series (B.1) to obtain an explicit series solution for $x(p)$ that includes the summation of leading and up to three subsequent subleading orders of logarithms.

The RG-equation may be expressed in the following form:

$$
\left(\frac{\partial}{\partial L}+\beta(x) \frac{\partial}{\partial x}\right) \sum_{n=0}^{\infty} \sum_{m=0}^{n} T_{n, m} x^{n+1} L^{m}=0,
$$

where $x \equiv x(\mu)$ and $L \equiv \log \left(\mu^{2} / p^{2}\right)$. We note from eq. (B.1) that when $L$ is equal to zero, $\mu^{2}$ is equal to $p^{2}$ and $x(\mu)=x(p)$. This initial condition implies that the series coefficients not involving logarithms satisfy the relations

$$
T_{0,0}=1 \quad T_{k, 0}=0 \quad(k \neq 0)
$$

Consider first the one-loop case in which $\beta(x)=-\beta_{0} x^{2}$. One then finds that the aggregate coefficient of $x^{p} L^{p-k} \quad(k \geq 2)$ on the left hand side of eq. (B.6) must vanish:

$$
(p-k+1) T_{p-1, p-k+1}-\beta_{0}(p-1) T_{p-2, p-k}=0
$$

Thus if $T_{k-2,0}=0$, as is the case for $k \geq 3$, then the recursion relation (B.8) guarantees that all coefficients $T_{p+k-2, p}$ within the series will vanish when $k>2$. Consequently, the only surviving coefficients of the series are those when $k=2$, i.e. the diagonal coefficients $T_{p, p}$, in which case we see from the series (B.1) that to one-loop order

$$
x(p)=x(\mu) \sum_{n=0}^{\infty} T_{n, n} x^{n}(\mu) \log ^{n}\left(\mu^{2} / p^{2}\right) .
$$


We also see from eqs. (B.7) and (B.8) that

$$
T_{n, n}=\beta_{0} T_{n-1, n-1}=\beta_{0}^{n} .
$$

Thus, eq. (B.9) is seen to be a geometric series whose explicit sum recovers the result (B.3).

Consider now the full $\beta$-function (5.4) for which the first four coefficients $\left\{\beta_{0}, \beta_{1}, \beta_{2}, \beta_{3}\right\}$ are known for QCD applications in the $\overline{M S}$ scheme [20]. Upon substitution of the $\beta$-function (5.4) into the left-hand side of the RG equation (B.6), we obtain the following recursion relations from the vanishing of the aggregate coefficients of $x^{p} L^{p-2}, x^{p} L^{p-3}, x^{p} L^{p-4}$, and $x^{p} L^{p-5}$, respectively:

$$
\begin{gathered}
T_{p, p}=\beta_{0} T_{p-1, p-1} \\
0=(p-2) T_{p-1, p-2}-\beta_{0}(p-1) T_{p-2, p-3}-\beta_{1}(p-2) T_{p-3, p-3} \\
0=(p-3) T_{p-1, p-3}-\beta_{0}(p-1) T_{p-2, p-4}-\beta_{1}(p-2) T_{p-3, p-4}-\beta_{2}(p-3) T_{p-4, p-4} \\
0=(p-4) T_{p-1, p-4}-\beta_{0}(p-1) T_{p-2, p-5}-\beta_{1}(p-2) T_{p-3, p-5} \\
-\beta_{2}(p-3) T_{p-4, p-5}-\beta_{3}(p-4) T_{p-5, p-5} .
\end{gathered}
$$

To make use of these recursion relations, we first re-organize the series (B.1) into the following form $\left[x \equiv x(\mu), L \equiv \log \left(\mu^{2} / p^{2}\right)\right]$

$$
x(p)=\sum_{n=0}^{\infty} S_{n}(x L) x^{n+1}, \quad S_{n}(x L)=\sum_{k=n}^{\infty} T_{k, k-n}(x L)^{k-n} .
$$

Since $T_{0,0}=1$, the recursion relation (B.11) implies that

$$
S_{0}(x L)=\sum_{k=0}^{\infty} T_{k, k}(x L)^{k}=1 /\left(1-\beta_{0} x L\right) .
$$

To evaluate the subsequent summations $S_{n}(u)$, where $n \geq 1$ and $u \equiv x L$, consider first the recursion relation (B.12). If we multiply this expression by $u^{p-3}$ and sum over $p$ from $p=3$ to $\infty$, we obtain

$$
\begin{aligned}
& \sum_{p=3}^{\infty}(p-2) T_{p-1, p-2} u^{p-3}-\beta_{0} \sum_{p=3}^{\infty}(p-1) T_{p-2, p-3} u^{p-3} \\
& -\beta_{1} \sum_{p=3}^{\infty}(p-2) T_{p-3, p-3} u^{p-3}=0 .
\end{aligned}
$$

Using the definitions (B.15) for $S_{0}$ and $S_{1}$, we find that eq. (B.17) is the following first order linear differential equation for $S_{1}(u)$ :

$$
\left(1-\beta_{0} u\right) \frac{d S_{1}}{d u}-2 \beta_{0} S_{1}=\beta_{1}\left[u \frac{d S_{0}}{d u}+S_{0}\right],
$$

where $S_{0}(u)$ is given by eq. (B.16) and where $S_{1}(0)=T_{1,0}=0$ by eqs. (B.7) and (B.15). The solution to this differential equation is

$$
S_{1}(u)=-\frac{\beta_{1}}{\beta_{0}} \frac{\log \left(1-\beta_{0} u\right)}{\left(1-\beta_{0} u\right)^{2}} .
$$


Similarly, we can multiply the recursion relations (B.13) and (B.14) by $u^{p-4}$ and $u^{p-5}$, respectively, and then by summing these respective equations from $p=4$ and $p=5$ to infinity. Using the definitions (B.15) for $S_{n}(u)$, we then obtain the following differential equations:

$$
\begin{gathered}
\left(1-\beta_{0} u\right) \frac{d S_{2}}{d u}-3 \beta_{0} S_{2}=\beta_{1}\left(u \frac{d S_{1}}{d u}+2 S_{1}\right)+\beta_{2}\left(u \frac{d S_{0}}{d u}+S_{0}\right), \\
\left(1-\beta_{0} u\right) \frac{d S_{3}}{d u}-4 \beta_{0} S_{3}=\beta_{1}\left(u \frac{d S_{2}}{d u}+3 S_{2}\right)+\beta_{2}\left(u \frac{d S_{1}}{d u}+2 S_{1}\right)+\beta_{3}\left(u \frac{d S_{0}}{d u}+S_{0}\right)
\end{gathered}
$$

with initial conditions (B.7): $S_{2}(0)=T_{2,0}=0, \quad S_{3}(0)=T_{3,0}=0$. The solution for $S_{2}(u)$ is then found to be

$$
\begin{aligned}
S_{2}(u) & =\left(\frac{\beta_{1}^{2}}{\beta_{0}^{2}}-\frac{\beta_{2}}{\beta_{0}}\right)\left[\left(1-\beta_{0} u\right)^{-2}-\left(1-\beta_{0} u\right)^{-3}\right] \\
& -\left(\frac{\beta_{1}}{\beta_{0}}\right)^{2}\left(1-\beta_{0} u\right)^{-3}\left[\log \left(1-\beta_{0} u\right)-\log ^{2}\left(1-\beta_{0} u\right)\right],
\end{aligned}
$$

and the corresponding solution for $S_{3}$ is

$$
\begin{aligned}
S_{3}(u) & =\left(-\frac{\beta_{3}}{2 \beta_{0}}+\frac{\beta_{1} \beta_{2}}{\beta_{0}^{2}}-\frac{\beta_{1}^{3}}{2 \beta_{0}^{3}}\right)\left(1-\beta_{0} u\right)^{-2} \\
& +\left(\frac{\beta_{1}^{3}}{\beta_{0}^{3}}-\frac{\beta_{1} \beta_{2}}{\beta_{0}^{2}}\right)\left(1-\beta_{0} u\right)^{-3} \\
& +\left(\frac{2 \beta_{1} \beta_{2}}{\beta_{0}^{2}}-\frac{2 \beta_{1}^{3}}{\beta_{0}^{3}}\right)\left(1-\beta_{0} u\right)^{-3} \log \left(1-\beta_{0} u\right) \\
& +\left(\frac{\beta_{3}}{2 \beta_{0}}-\frac{\beta_{1}^{3}}{2 \beta_{0}^{3}}\right)\left(1-\beta_{0} u\right)^{-4} \\
& +\left(\frac{2 \beta_{1}^{3}}{\beta_{0}^{3}}-\frac{3 \beta_{1} \beta_{2}}{\beta_{0}^{2}}\right)\left(1-\beta_{0} u\right)^{-4} \log \left(1-\beta_{0} u\right) \\
& +\frac{\beta_{1}^{3}}{\beta_{0}^{3}}\left(1-\beta_{0} u\right)^{-4}\left(\frac{5}{2} \log ^{2}\left(1-\beta_{0} u\right)-\log ^{3}\left(1-\beta_{0} u\right)\right) .
\end{aligned}
$$

Upon substitution of expressions (B.16), (B.17), (B.22) and (B.23) for $\left\{S_{0}, S_{1}, S_{2}\right.$ and $\left.S_{3}\right\}$ into eq. (B.15), or alternatively, into eq. (5.9), we obtain eq. (5.14) for the RG invariant effective couplant $x(p)$.

\section{References}

[1] E. C. G. Stueckelberg and A. Peterman, Helv. Phys. Acta 26 (1953) 499; N. N. Bogoliubov and D. V. Shirkov, Introduction to the Theory of Quantized Fields (Interscience, NY, 1959); M. Gell-Mann and F. Low, Phys. Rev. 95 (1954) 1300; C. Callan, Phys. Rev. D2 (1970) 1541; K. Symanzik, Comm. Math. Phys. 18 (1970) 227; S. Weinberg, Phys. Rev. D 8 (1973) 3497; A. Peterman, Phys. Rep. 53 C (1979) 157; P. M. Stevenson, Ann. Phys. 132 (1981) 383.

[2] G. 't Hooft, Nucl. Phys. B 61 (1973) 455; J. C. Collins and A. J. Macfarlane, Phys. Rev. D 10 (1974) 1201.

[3] G. 't Hooft and M. Veltman, Nucl. Phys. B 44 (1972) 189; J. F. Ashmore, Nuovo Cim. Lett. 4 (1972) 289; C. G. Bollini and J. J. Giambiagi, Nuovo Cim. B 12 (1972) 20. 
[4] Abdus Salam and J. Strathdee, Nucl. Phys. B 90 (1975) 203; J. Dowker and R. Critchley, Phys. Rev. D 13 (1976) 3224; S. Hawking, Comm. Math. Phys. 55 (1977) 133.

[5] D. G. C. McKeon and T. N. Sherry, Phys. Rev. D 35 (1987) 3854; D. G. C. McKeon and T. N. Sherry, Ann. Phys. 218 (1992) 325.

[6] L. Culumovic, D. G. C. McKeon, and T. N. Sherry, Ann. Phys. 197 (1989) 94.

[7] L. Culumovic, M. Leblanc, R. B. Mann, D. G. C. McKeon, and T. N. Sherry, Phys. Rev. D 41 (1990) 514 .

[8] V. Elias and D. G. C. McKeon, hep-th/0209151.

[9] D. G. C. McKeon, Int. J. Theor. Phys. 37 (1998) 817.

[10] M. R. Ahmady, F. A. Chishtie, V. Elias, A. H. Fariborz, N. Fattahi, D.G.C. McKeon, T. N. Sherry and T. G. Steele, Phys. Rev. D 66 (2002) 014010.

[11] B. Kastening, Phys. Lett. B 283 (1992) 287.

[12] J. -M. Chung and B. K. Chung, Phys. Rev. D 60 (1999) 105001.

[13] W. Dittrich and M. Reuter, Effective Actions in Quantum Electrodynamics (SpringerVerlag, Berlin, 1985) Chap. 8.

[14] V. Novikov, M. Shifman, A. I. Vainshtein, and V. Zakharov, Nucl. Phys. B 229 (1983) 381.

[15] D. R. T. Jones, Phys. Lett. B 123 (1983) 45; V. Elias, J. Phys. G 27 (2001) 217.

[16] G. 't Hooft, Phys. Rev. D 14 (1976) 3432.

[17] R. M. Corless, G. H. Gonnet, D. E. G. Hare, D. J. Jeffrey and D. E. Knuth, Adv. Comput. Math. 5 (1996) 329.

[18] G. 't Hooft, Nucl. Phys. B 62 (1973) 444; L. Abbott, Nucl. Phys. B 185 (1981) 189. 1201.

[19] Matinyen and K. Savvidy, Nucl. Phys. B 134 (1978) 539; A. J. Buras, Rev. Mod. Phys. 52 (1980) 199.

[20] D. J. Gross and F. Wilczek, Phys. Rev. Lett. 30 (1973) 1343; H. D. Politzer, Phys. Rev. Lett. 30 (1973) 1346; W. E. Caswell, Phys. Rev. Lett. 33 (1974) 244; D. R. T. Jones, Nucl. Phys. B 75 (1974) 531; E. S. Egorian, O. V. Tarasov, Theor. Mat. Fiz. 41 (1979) 26; O. V. Tarasov, A. A. Vladimirov, and A. Yu. Zharkov, Phys. Lett. B 93 (1980) 429; S. A. Larin and J. A. M. Vermaseren, Phys. Lett. B 303 (1993) 334; T. van Ritbergen, J. A. M. Vermaseren, and S. A. Larin, Phys. Lett. B 400 (1997) 379.

[21] ALEPH Collaboration, R. Barate et al. Eur. Phys. J. C 4 (1998) 409; T. G. Steele and V. Elias, Mod. Phys. Lett. A 13 (1998) 3151; G. Cvetic and T. Lee, Phys. Rev. D 64 (2001) 014030; C. J. Maxwell and A. Mirjalili, Nucl. Phys. B 611 (2001) 423.

[22] G. 't Hooft, in The Whys of Subnuclear Physics, A. Zichichi, ed. (Plenum, NY, 1979) pp. 943-971. 


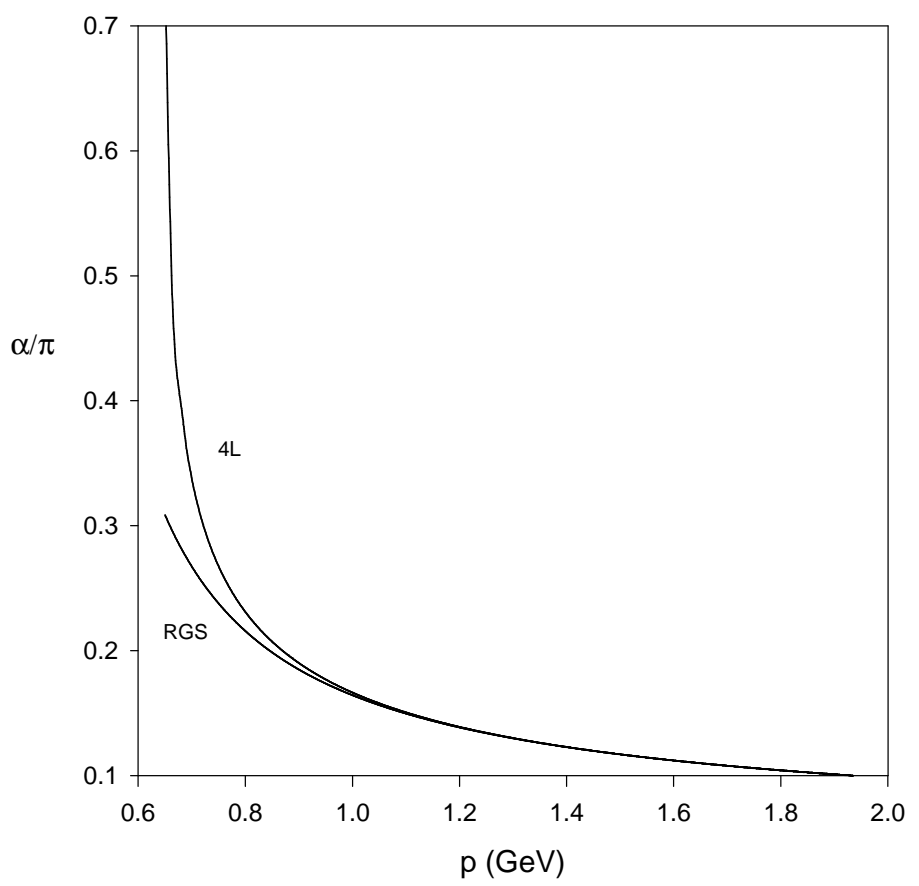

Figure 1: Comparison of the true four-loop (4L) effective coupling constant, as obtained via integration of the four-loop $\beta$-function, to the coupling constant obtained in eq. (5.14) via summation of successively subleading logarithmic terms (RGS). 


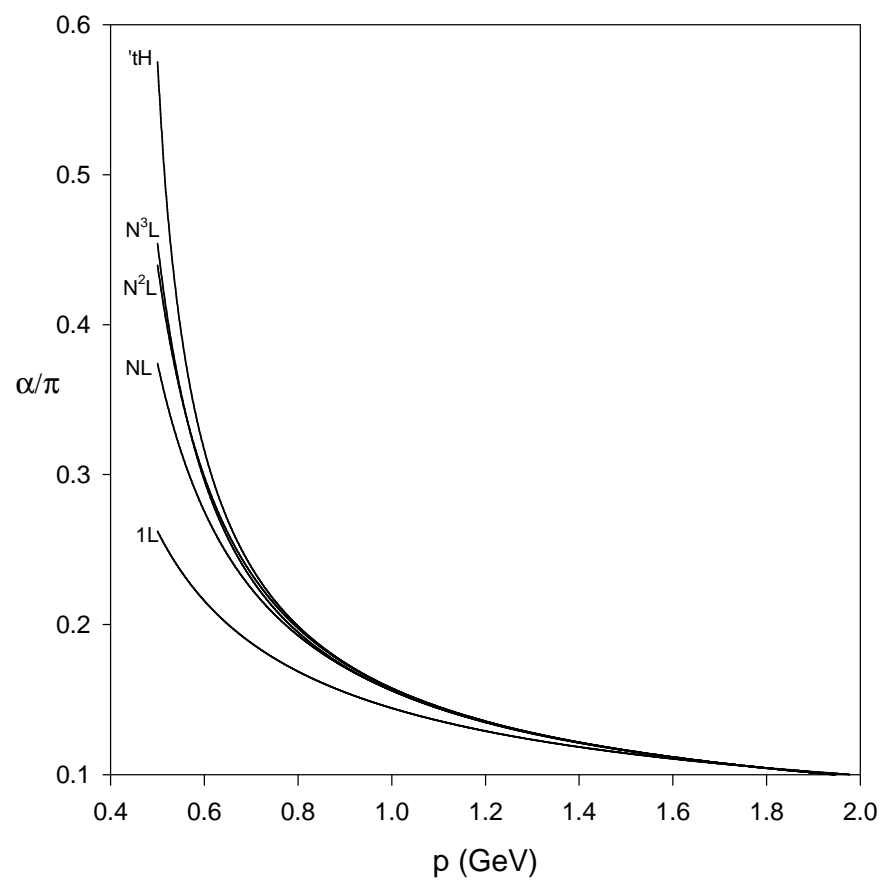

Figure 2: Comparison of successive subleading approximations to the 't Hooft scheme effective couplant, as discussed in the text, to the exact evolution of the 't Hooft scheme ('tH) couplant. 